\title{
PARALLEL SPINORS AND CONNECTIONS WITH SKEW-SYMMETRIC TORSION IN STRING THEORY*
}

\author{
THOMAS FRIEDRICH ${ }^{\dagger}$ AND STEFAN IVANOV $\ddagger$
}

\begin{abstract}
We describe all almost contact metric, almost hermitian and $G_{2}$-structures admitting a connection with totally skew-symmetric torsion tensor, and prove that there exists at most one such connection. We investigate its torsion form, its Ricci tensor, the Dirac operator and the $\nabla$ parallel spinors. In particular, we obtain partial solutions of the type $I I$ string equations in dimension $n=5,6$ and 7 .
\end{abstract}

1. Introduction. Linear connections preserving a Riemannian metric with totally skew-symmetric torsion recently became a subject of interest in theoretical and mathematical physics. For example, the target space of supersymmetric sigma models with Wess-Zumino term carries a geometry of a metric connection with skew-symmetric torsion [23, 34, 35] (see also [42] and references therein). In supergravity theories, the geometry of the moduli space of a class of black holes is carried out by a metric connection with skew-symmetric torsion [27]. The geometry of NS-5 brane solutions of type II supergravity theories is generated by a metric connection with skew-symmetric torsion $[44,45,43]$. The existence of parallel spinors with respect to a metric connection with skew-symmetric torsion on a Riemannian spin manifold is of importance in string theory, since they are associated with some string solitons (BPS solitons) [43]. Supergravity solutions that preserve some of the supersymmetry of the underlying theory have found many applications in the exploration of perturbative and non-perturbative properties of string theory. An important example is the AdS/CFT correspondence, also known as the Maldacena conjecture, which conjectures duality between the supersymmetric background and a certain superconformal field theory $[39,32,51]$.

In type II string theory one investigates manifolds $N^{k} \times M^{10-k}$, where $N^{k}$ is a $k$-dimensional space-time and $M^{10-k}$ is a Riemannian manifold equipped with an additional structure. Indeed, the basic model is a 5 -tuple $\left(M^{n}, g, H, \Phi, \Psi\right)$, where $g$ is a Riemannian metric, $H$ is a 3 -form, $\Phi$ is the socalled dilation function, and $\Psi$ is a spinor field. The string equations, which are a generalization of the Einstein equations, can be written in the following form (see [48]):

$$
\operatorname{Ric}_{i j}^{g}-\frac{1}{4} H_{i m n} H_{j m n}+2 \cdot \nabla_{i}^{g} \partial_{j} \Phi=0, \quad \delta\left(e^{-2 \Phi} H\right)=0 .
$$

The field equations are supplemented with the so-called Killing spinor equations

$$
\left.\left(\nabla_{X}^{g}+\frac{1}{4} X\right\lrcorner H\right) \cdot \Psi=0, \quad(2 \cdot d \Phi-H) \cdot \Psi=0 .
$$

${ }^{*}$ Received February 17, 2002; accepted for publication February 26, 2002. Supported by the SFB 288 of the DFG. S.I. is a member of the EDGE,Research Training Network HPRN-CT-2000-00101, supported by the European Human Potential Programme.S.I. thanks the Humboldt University Berlin and ICTP, Trieste for the support and excellent enviroments.

†Humboldt-Universität zu Berlin, Institut für Mathematik, 10099 Berlin, Germany (friedric@mathematik.hu-berlin.de).

$\ddagger$ University of Sofia "St. Kl. Ohridski", Faculty of Mathematics and Informatics, blvd. James Bourchier 5, 1164 Sofia, Bulgaria (ivanovsp@fmi.uni-sofia.bg). 
Sometimes one requires that the 3 -form $H$ is closed, $d H=0$, and solutions of this type are then called strong. The first of the Killing spinor equations suggests that the 3-form $H$ should be the torsion form of a metric connection $\nabla$ with totally skewsymmetric torsion tensor $T=H$. Then the string equations and the Killing spinor equations can be written as follows (see [36]) :

$$
\begin{gathered}
\left.\operatorname{Ric}^{\nabla}+\frac{1}{2} \delta(T)+2 \cdot \nabla^{g} d \Phi=0, \quad \delta(T)=2 \cdot d \Phi^{\#}\right\lrcorner T \\
\nabla \Psi=0, \quad(2 \cdot d \Phi-T) \cdot \Psi=0 .
\end{gathered}
$$

$\mathrm{Ric}^{\nabla}$ is the Ricci tensor of the metric connection $\nabla, \nabla^{g}$ is the Levi-Civita connection of the metric $g$ and $d \Phi^{\#}$ denotes the vector field dual to the 1-form $d \Phi$. If the dilation is constant, then the string equations are equivalent to the condition that the Ricci tensor of the connection vanishes $[36], \operatorname{Ric}^{\nabla}=0$, and the Killing spinor equations become

$$
\nabla \Psi=0, \quad T \cdot \Psi=0 .
$$

In particular, the spinor field is Riemannian harmonic. More general, the Riemannian Dirac operator $D^{g}$ acts on a $\nabla$-parallel spinor field via the formula

$$
D^{g} \Psi+\frac{3}{4} T \cdot \Psi=0
$$

The number of preserved supersymmetries depends essentially on the number of $\nabla$-parallel spinors. In this paper we investigate solutions of all these field equations in the case of constant dilation in dimensions $n=5,6,7$. In dimension 7 we derive a topological obstruction for the existence of such solutions (Remark 5.5).

A spinor parallel with respect to the Levi-Civita connection on a Riemannian manifold $M^{n}$ restricts its holonomy group (see [33, 50] and [40]). In a similar way a $\nabla$-parallel spinor field reduces the structure group of the frame bundle. Conversely, let us start with a (non-integrable) $G$-structure on a Riemannian manifold and ask the question whether or not there exists a $G$-connection with a totally skew-symmetric torsion and at least one parallel spinor field. In dimension 3 the stabilizer of Spin(3) is trivial and therefore the connection is flat. It is well known (see e.g. [47]) that in this case $\left(M^{3}, g, \nabla, T\right)$ carries (locally) a structure of a compact Lie group, $g$ is a biinvariant metric and $\nabla$ is the invariant connection with torsion given by the Lie bracket. In particular, on $S U(2)$ there exists at least one $\nabla$-parallel spinor.

The 4-dimensional case was investigated in earlier papers. The restricted holonomy group of $\nabla$ should be contained in $\mathrm{SU}(2)$ (see [43]) and this is equivalent to the local existence of a HKT structure, i.e., a hyperhermitian structure that is parallel with respect to $\nabla$ (see [36]). Surprisingly, the geometry of $\nabla$ depends on the type of the parallel spinor (see [10]). If $M^{4}$ is compact, then the holonomy of $\nabla$ is contained in $\mathrm{SU}(2)$ if and only if $M^{4}$ is either a Calabi-Yau manifold or a Hopf surface (see [36]). We note that there exist Hopf surfaces that do not admit any (global) hyperhermitian structure although the holonomy of $\nabla$ is contained in $\mathrm{SU}(2)$ (see [24], [36]). These Hopf surfaces do not admit any $\nabla$-parallel spinors (see [10]), which shows that in the non-simply connected compact case the holonomy condition is not sufficient for the 
existence of $\nabla$-parallel spinors.

In higher dimensions we fix a subgroup $G$ of $\operatorname{Spin}(n)$ preserving a spinor $\Psi_{0}$ as well as a geometric $G$-structure on a Riemannian manifold $\left(M^{n}, g\right)$. Then

a) we describe the set of all $G$-connections with totally skew-symmetric torsion for any geometric type of $G$-structures. In particular, we decide whether or not a $G$-structure admits a connection with totally skew-symmetric torsion $T$ and derive a formula for the torsion;

b) We use the parallel spinor $\Psi_{0}$ and the algebraic properties of the Clifford multiplication in special dimensions in order to derive the field equation;

c) We study the space of all $\nabla$-parallel spinors and compare it to the space of $\nabla$-harmonic spinors. In particular, we decide whether or not there exist $\nabla$-parallel spinor fields $\Psi$ such that $T \cdot \Psi=0$.

In the second and third step we need the Schrödinger-Lichnerowicz-formula for a metric connection with totally skew-symmetric torsion. In particular, we prove this formula in full generality, including the computation of the curvature term.

We will give a complete answer in dimension $n=7$ and for $G_{2}$-structures. In odd dimensions (almost contact metric structures) and in even dimensions (almost hermitian structures) we solve the first problem. However, these geometric structures reduce the structure group of the frame bundle only to the subgroup $U(k)$, which does not coincide with the isotropy group of a spinor. Consequently, the connection $\nabla$ adapted to the geometric structure under consideration does not admit a $\nabla$-parallel spinor automatically and we obtain a further curvature condition for the existence of such spinors. We investigate this condition and prove vanishing theorems for $\nabla$-harmonic spinors.

2. The curvature of connections with totally skew-symmetric torsion. In this section we recall some notions concerning the curvature of a metric connection with totally skew-symmetric torsion from [36]. Let $\left(M^{n}, g, \nabla, T\right)$ be an $n$-dimensional Riemannian manifold with a metric connection $\nabla$ of totally skew-symmetric torsion $T$. The torsion tensor measures the difference between the connection $\nabla$ and the Levi-Civita connection $\nabla^{g}$ :

$$
g\left(\nabla_{X} Y, Z\right)=g\left(\nabla_{X}^{g} Y, Z\right)+\frac{1}{2} T(X, Y, Z) .
$$

Let us fix some notation. The differential of an exterior form $\alpha$ is given by the formula

$$
d \alpha=\sum_{i=1}^{n} e_{i} \wedge \nabla_{e_{i}}^{g} \alpha
$$

The codifferential of the form $\alpha$ can be calculated using either the Levi-Civita connection $\nabla^{g}$ or the connection $\nabla$ :

$$
\left.\left.\delta^{g}(\alpha)=-\sum_{i=1}^{n} e_{i}\right\lrcorner \nabla_{e_{i}}^{g} \alpha, \quad \delta^{\nabla}(\alpha)=-\sum_{i=1}^{n} e_{i}\right\lrcorner \nabla_{e_{i}} \alpha .
$$

On the 3-form $T$, the two codifferentials coincide :

$$
\delta^{g}(T)=\delta^{\nabla}(T)
$$


This formula is a consequence of the assumption that $\nabla$ has a totally skew-symmetric torsion tensor. Let us introduce the 4-form $\sigma^{T}$

$$
\begin{aligned}
\sigma^{T}(X, Y, Z, V) & :=g(T(X, Y), T(Z, V))+g(T(Y, Z), T(X, V))+g(T(Z, X), T(Y, V)) \\
& \left.\left.=\frac{1}{2} \sum_{i=1}^{n}\left(e_{i}\right\lrcorner T\right) \wedge\left(e_{i}\right\lrcorner T\right)(X, Y, Z, V) .
\end{aligned}
$$

Then the exterior derivative $d T$ of the torsion tensor $T$ is given in terms of $\nabla$ by the following formula (see e.g.[36])

$$
d T(X, Y, Z, V)=\sigma_{X Y Z}\left\{\left(\nabla_{X} T\right)(Y, Z, V)\right\}-\left(\nabla_{V} T\right)(X, Y, Z)+2 \sigma^{T}(X, Y, Z, V),
$$

where $\sigma_{X Y Z}$ denotes the cyclic sum over $X, Y, Z$. Moreover, the curvature tensors of the Levi-Civita connection and the connection $\nabla$ are related via the formula

$$
\begin{aligned}
R^{g}(X, Y, Z, V)= & R^{\nabla}(X, Y, Z, V)-\frac{1}{2}\left(\nabla_{X} T\right)(Y, Z, V)+\frac{1}{2}\left(\nabla_{Y} T\right)(X, Z, V) \\
& -\frac{1}{4} g(T(X, Y), T(Z, V))-\frac{1}{4} \sigma^{T}(X, Y, Z, V) .
\end{aligned}
$$

The first Bianchi identity for $\nabla$ can be written in the form

$$
\sigma_{X Y Z} R^{\nabla}(X, Y, Z, V)=d T(X, Y, Z, V)-\sigma^{T}(X, Y, Z, V)+\left(\nabla_{V} T\right)(X, Y, Z)
$$

and the difference of the Ricci tensors involves the codifferential of $T$ :

$$
\operatorname{Ric}^{g}(X, Y)=\operatorname{Ric}^{\nabla}(X, Y)+\frac{1}{2} \delta^{g}(T)(X, Y)-\frac{1}{4} \sum_{i=1}^{n} g\left(T\left(e_{i}, X\right), T\left(Y, e_{i}\right)\right) .
$$

In particular, the skew-symmetric part of the Ricci tensor $\operatorname{Ric}^{\nabla}$ of $\nabla$ is given by the codifferential of the torsion tensor only :

$$
\operatorname{Ric}^{\nabla}(X, Y)-\operatorname{Ric}^{\nabla}(Y, X)=-\delta^{g}(T)(X, Y) .
$$

We denote the scalar curvature of $\nabla$ by $\mathrm{Scal}^{\nabla}$, i.e.,

$$
\mathrm{Scal}^{\nabla}=\sum_{i, j=1}^{n} R^{\nabla}\left(e_{i}, e_{j}, e_{j}, e_{i}\right)
$$

3. The Schrödinger-Lichnerowicz-formula for connections with totally skew-symmetic torsion. Consider an $n$-dimensional Riemannian spin manifold $\left(M^{n}, g, \nabla, T\right)$ with a metric connection $\nabla$ of totally skew-symmetric torsion $T$ and denote by $\Sigma M^{n}$ the spinor bundle. The Dirac operator $D$ depending on the connection $\nabla$ is defined by

$$
D \Psi=\sum_{i=1}^{n} e_{i} \cdot \nabla_{e_{i}} \Psi, \quad \Psi \in \Sigma M^{n}
$$

where $e_{1} \ldots e_{n}$ is an orthonormal basis. The Dirac operator $D$ is a formally selfadjoint operator since the torsion of the connection is totally skew-symmetric (see [21]). In case of a Riemannian manifold the well-known Schrödinger-Lichnerowicz-formula expresses the square of the Dirac operator with respect to the Levi-Civita connection 
by the spinorial Laplace operator and some curvature term (see $[46,14])$. In the articles $[5,1]$, a generalization of this formula for connections with arbitrary torsion is indicated. For connections with totally skew-symmetric torsion we shall derive the curvature term and prove the following explicit formula :

Theorem 3.1. (S-L-Formula) Let $\left(M^{n}, g, \nabla, T\right)$ be an n-dimensional Riemannian spin manifold with a metric connection $\nabla$ of totally skew-symmetric torsion $T$. Then, for any spinor field $\Psi$, the formula

$$
\left.D^{2} \Psi=\nabla^{*} \nabla \Psi+\frac{3}{4} d T \cdot \Psi-\frac{1}{2} \sigma^{T} \cdot \Psi+\delta^{g}(T) \cdot \Psi-\sum_{k=1}^{n} e_{k}\right\lrcorner T \cdot \nabla_{e_{k}} \Psi+\frac{1}{4} \mathrm{Scal}^{\nabla} \cdot \Psi
$$

holds, where $\nabla^{*} \nabla$ is the Laplacian of $\nabla$ acting on spinors by

$$
\nabla^{*} \nabla \Psi=-\sum_{i=1}^{n} \nabla_{e_{i}} \nabla_{e_{i}} \Psi+\nabla_{\nabla_{e_{i}}^{g} e_{i}} \Psi .
$$

Proof. At a fixed point $p \in M^{n}$ we choose an orthonormal basis $e_{1}, \ldots, e_{n}$ such that $\left(\nabla_{e_{i}} e_{j}\right)_{p}=0$ and $\left[e_{i}, e_{j}\right]_{p}=-T\left(e_{i}, e_{j}\right)_{p}$. Then the vector field $e_{i}$ is $\nabla^{g}$-parallel in the direction of $e_{i}$ at the point $p:\left(\nabla_{e_{i}}^{g} e_{i}\right)_{p}=0$. We calculate

$$
\begin{aligned}
D^{2} \Psi & =\sum_{i, j=1}^{n} e_{i} \nabla_{e_{i}} e_{j} \nabla_{e_{j}} \Psi \\
& \left.=-\sum_{i=1}^{n} \nabla_{e_{i}} \nabla_{e_{i}} \Psi+\sum_{i, j=1}^{n} R^{\nabla}\left(e_{i}, e_{j}\right) e_{i} \cdot e_{j} \cdot \Psi-\sum_{i=1}^{n} e_{i}\right\lrcorner T \cdot \nabla_{e_{i}} \Psi \\
& \left.=\nabla^{*} \nabla \Psi+\frac{1}{2} \sum_{i<j, k<l} R^{\nabla}\left(e_{i}, e_{j}, e_{k}, e_{l}\right) e_{i} \cdot e_{j} \cdot e_{k} \cdot e_{l} \cdot \Psi-\sum_{i=1}^{n} e_{i}\right\lrcorner T \cdot \nabla_{e_{i}} \Psi .
\end{aligned}
$$

The curvature term in the latter equation can be written in the form

$$
\hat{\sigma}\left(R^{\nabla}\right) \cdot \Psi-\sum_{j<k}\left[\operatorname{Ric}^{\nabla}\left(e_{j}, e_{k}\right)-\operatorname{Ric}^{\nabla}\left(e_{k}, e_{j}\right)\right] e_{j} \cdot e_{k} \cdot \Psi+\frac{1}{2} \operatorname{Scal}^{\nabla} \cdot \Psi,
$$

where $\hat{\sigma}\left(R^{\nabla}\right)$ is the 4 -form given by

$$
\hat{\sigma}\left(R^{\nabla}\right)(X, Y, Z, V)=\sigma_{X Y Z}\left\{R^{\nabla}(X, Y, Z, V)-R^{\nabla}(V, X, Y, Z)\right\} .
$$

Using the formulas for $d T$ as well as the formula comparing the curvature tensors $R^{g}$ and $R^{\nabla}$ we obtain

$$
\hat{\sigma}\left(R^{\nabla}\right)=\frac{3}{2} d T-\sigma^{T} .
$$

Inserting the latter formula as well as the formula for the skew-symmetric part of the Ricci tensor into the expression for $D^{2} \Psi$ yields the desired formula.

COROLLARY 3.2. Let $\Psi$ be a parallel spinor with respect to $\nabla$. Then the following formulas hold:

$$
\frac{3}{4} d T \cdot \Psi-\frac{1}{2} \sigma^{T} \cdot \Psi+\frac{1}{2} \delta^{g}(T) \cdot \Psi+\frac{1}{4} \operatorname{Scal}^{\nabla} \cdot \Psi=0,
$$




$$
\left.\left(\frac{1}{2} X\right\lrcorner d T+\nabla_{X} T\right) \cdot \Psi-\operatorname{Ric}^{\nabla}(X) \cdot \Psi=0 .
$$

Proof. The first formula follows directly from Theorem 3.1. We prove the second one by contracting the well-known formula

$$
0=\nabla \nabla \Psi=\sum_{i, j=1}^{n} R^{\nabla}\left(e_{i}, e_{j}\right) \cdot e_{i} \cdot e_{j} \cdot \Psi
$$

and using the formulas relating the symmetrization of the curvature tensor $R^{\nabla}$ and the derivative of the torsion form $T$.

The next formula compares the action of the Dirac operator with the action of the torsion form on spinors.

TheOREM 3.3. Let $\left(M^{n}, g, \nabla, T\right)$ be an $n$-dimensional Riemannian spin manifold with a metric connection $\nabla$ of totally skew-symmetric torsion. Then

$$
\left.D T+T D=d T+\delta^{g}(T)-2 \cdot \sigma^{T}-2 \sum_{i=1}^{n} e_{i}\right\lrcorner T \cdot \nabla_{e_{i}} .
$$

Proof. The proof is similar to the proof of Theorem 3.1.

Let us apply Theorem 3.1 and Theorem 3.3 in case of a $\nabla$-harmonic spinor field $\Psi$ on a compact manifold $M^{n}$. Since $\delta^{g}(T)$ is a 2 -form, the real part of the hermitian product $\left(\delta^{g}(T) \cdot \Psi, \Psi\right)$ vanishes. Moreover, the Dirac operator $D$ is symmetric. Combining Theorem 3.1 and Theorem 3.3 we obtain the condition

$$
\int_{M^{n}}\left(\|\nabla \Psi\|^{2}+\frac{1}{4}(d T \cdot \Psi, \Psi)+\frac{1}{2}\left(\sigma^{T} \cdot \Psi, \Psi\right)+\frac{1}{4} \operatorname{Scal}^{\nabla} \cdot\|\Psi\|^{2}\right)=0 .
$$

This formula proves the following vanishing theorem.

THEOREM 3.4. Let $\left(M^{n}, g, \nabla, T\right)$ be a compact Riemannian spin manifold with a metric connection $\nabla$ of totally skew-symmetric torsion $T$. Suppose, moreover, that the eigenvalues of the endomorphism $d T+2 \cdot \sigma^{T}+\mathrm{Scal}^{\nabla}$ acting on spinors are non-negative. Then any $\nabla$-harmonic spinor field is $\nabla$-parallel. In case the eigenvalues of the endomorphism are positive, there are no $\nabla$-parallel spinor fields.

4. $G_{2}$-connections with totally skew-symmetric torsion. We study under which conditions a fixed $G$-structure on a Riemannian manifold admits an affine connection preserving the $G$-structure and having totally skew-symmetric torsion tensor. For this purpose we describe the different geometric types of $G$-structures from the point of view of gauge theory using a certain 1-form $\Gamma$ with values in the associated bundle of typical fibres $\mathfrak{s o}(n) / \mathfrak{g}$. This approach is completely equivalent to the classification of different geometric $G$-structures used in differential geometry and mainly based on the decomposition of the covariant derivative of the tensor related with the $G$-structure. The advantage of our approach is that the method applies even in cases where the $G$-structure is not defined by a tensor (see $[15,49]$ ). To begin with, let $\left(M^{n}, g\right)$ be an oriented Riemannian manifold and denote by $\mathcal{F}\left(M^{n}\right)$ its frame bundle. The Levi-Civita connection is a 1-form

$$
Z^{g}: T\left(\mathcal{F}\left(M^{n}\right)\right) \longrightarrow \mathfrak{s o}(n)
$$


with values in the Lie algebra $\mathfrak{s o}(n)$. Its torsion tensor vanishes. We fix a closed subgroup $G$ of the orthogonal group $S O(n)$. A $G$-structure on $M^{n}$ is a $G$-subbundle $\mathcal{R} \subset \mathcal{F}\left(M^{n}\right)$. We decompose the Lie algebra $\mathfrak{s o}(n)$ into the subalgebra $\mathfrak{g}$ and its orthogonal complement $\mathfrak{m}$ :

$$
\mathfrak{s o}(n)=\mathfrak{g} \oplus \mathfrak{m} .
$$

In a similar way we decompose the restriction of the 1-form $Z^{g}$

$$
\left.Z^{g}\right|_{T(\mathcal{R})}=\tilde{Z} \oplus \Gamma .
$$

$\tilde{Z}$ is a connection in the principal $G$-bundle $\mathcal{R}$ and $\Gamma$ is a 1 -form with values in the associated bundle $\mathcal{R} \times{ }_{G} \mathfrak{m}$. The different geometric types of $G$-structures are defined by the irreducible $G$-components of the representation $\mathbb{R}^{n} \otimes \mathfrak{m}$. An arbitrary $G$-connection $Z$ differs from $\tilde{Z}$ by an 1 -form $\Sigma$ with values in the Lie algebra $\mathfrak{g}$,

$$
Z=Z^{g}-\Gamma+\Sigma
$$

and the corresponding covariant derivative $\nabla$ is given by the formula

$$
\nabla_{X} Y=\nabla_{X}^{g} Y-\Gamma(X)(Y)+\Sigma(X)(Y) .
$$

Since the Levi-Civita connection $\nabla^{g}$ is torsion free, the torsion tensor of $\nabla$ depends on $\Gamma$ and $\Sigma$ :

$T(X, Y, Z)=-g(\Gamma(X)(Y), Z)+g(\Gamma(Y)(X), Z)+g(\Sigma(X)(Y), Z)-g(\Sigma(Y)(X), Z)$.

$T$ is a 3 -form if and only if

$$
g(\Gamma(Y)(X), Z)+g(\Gamma(Z)(X), Y)=g(\Sigma(Z)(X), Y)+g(\Sigma(Y)(X), Z)
$$

holds. Now we introduce the following $G$-invariant maps:

$$
\begin{aligned}
& \Phi: \mathbb{R}^{n} \otimes \mathfrak{g} \rightarrow \mathbb{R}^{n} \otimes S^{2}\left(\mathbb{R}^{n}\right), \Phi(\Sigma)(X, Y, Z):=g(\Sigma(Z)(X), Y)+g(\Sigma(Y)(X), Z), \\
& \Psi: \mathbb{R}^{n} \otimes \mathfrak{m} \rightarrow \mathbb{R}^{n} \otimes S^{2}\left(\mathbb{R}^{n}\right), \Psi(\Gamma)(X, Y, Z):=g(\Gamma(Y)(X), Z)+g(\Gamma(Z)(X), Y) .
\end{aligned}
$$

Consequently, we proved the following

Proposition 4.1. A G-reduction $\mathcal{R} \subset \mathcal{F}\left(M^{n}\right)$ admits a $G$-connection $Z$ with a totally skew-symmetric torsion tensor $T$ if and only if $\Psi(\Gamma)$ is contained in the image of the homomorphism $\Phi$. In this case the set of all these connections $Z$ is an affine space over the vector space $\operatorname{ker}(\Phi)$.

We will use representation theory in order to study the diagramme

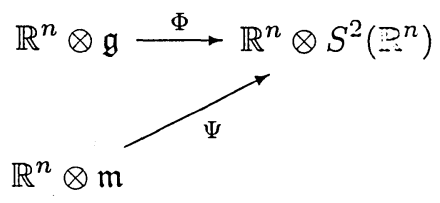

By splitting the $G$-representation $\mathbb{R}^{n} \otimes \mathfrak{m}$ into irreducible components we can decide whether or not the image of a certain component is contained in the image of $\Phi$. 
In this way we characterize the geometric $G$-structures admitting a $G$-connection $Z$ with a totally skew-symmetric torsion tensor. We will apply this general method to the subgroup $G_{2} \subset S O(7)$. Therefore, let us recall some notions of $G_{2}$-geometry in dimension seven. The group $G_{2}$ is the isotropy group of the 3-form in seven variables $\omega^{3}:=e_{1} \wedge e_{2} \wedge e_{7}+e_{1} \wedge e_{3} \wedge e_{5}-e_{1} \wedge e_{4} \wedge e_{6}-e_{2} \wedge e_{3} \wedge e_{6}-e_{2} \wedge e_{4} \wedge e_{5}+e_{3} \wedge e_{4} \wedge e_{7}+e_{5} \wedge e_{6} \wedge e_{7}$.

The 3-form $\omega^{3}$ corresponds to a real spinor $\Psi_{0} \in \Delta_{7}$ and, therefore, $G_{2}$ can be defined as the isotropy group of a non-trivial real spinor. We identify the Lie algebra of the group $S O(7)$ with the space of all 2-forms :

$$
\mathfrak{s o}(7)=\Lambda^{2}\left(\mathbb{R}^{7}\right)=\left\{\sum_{i<j} \omega_{i j} \cdot e_{i} \wedge e_{j}\right\} .
$$

The Lie algebra $\mathfrak{g}_{2}$ of the group $G_{2}$ is given by the equations

$$
\begin{aligned}
& \omega_{12}+\omega_{34}+\omega_{56}=0, \quad-\omega_{13}+\omega_{24}-\omega_{67}=0, \quad \omega_{14}+\omega_{23}+\omega_{57}=0, \\
& \omega_{16}+\omega_{25}-\omega_{37}=0, \quad \omega_{15}-\omega_{26}-\omega_{47}=0, \quad \omega_{17}+\omega_{36}+\omega_{45}=0, \\
& \omega_{27}+\omega_{35}-\omega_{46}=0 .
\end{aligned}
$$

The space $\mathbb{R}^{7}:=\Lambda_{7}^{1}$ is an irreducible $G_{2}$-representation, the 2-forms $\Lambda^{2}=\Lambda_{7}^{2} \oplus \Lambda_{14}^{2}$ split into two irreducible $G_{2}$-components :

$$
\begin{aligned}
\Lambda_{7}^{2} & \left.:=\left\{\alpha^{2} \in \Lambda^{2}: *\left(\omega^{3} \wedge \alpha^{2}\right)=2 \cdot \alpha^{2}\right\}=\{X\lrcorner \omega^{3}: X \in \mathbb{R}^{7}\right\} \\
\Lambda_{14}^{2} & :=\left\{\alpha^{2} \in \Lambda^{2}: *\left(\omega^{3} \wedge \alpha^{2}\right)=-\alpha^{2}\right\}=\mathfrak{g}_{2} .
\end{aligned}
$$

The space of 3 -forms $\Lambda^{3}=\Lambda_{1}^{3} \oplus \Lambda_{7}^{3} \oplus \Lambda_{27}^{3}$ decomposes into three irreducible $G_{2^{-}}$ components :

$$
\begin{aligned}
\Lambda_{1}^{3} & :=\left\{t \cdot \omega^{3}: t \in \mathbb{R}\right\} \\
\Lambda_{7}^{3} & \left.:=\left\{*\left(\omega^{3} \wedge \alpha^{1}\right): \alpha^{1} \in \Lambda^{1}\right\}=\{X\lrcorner * \omega^{3}: X \in \mathbb{R}^{7}\right\} \\
\Lambda_{27}^{3} & :=\left\{\alpha^{3} \in \Lambda^{3}: \alpha^{3} \wedge \omega^{3}=0, \alpha^{3} \wedge * \omega^{3}=0\right\} .
\end{aligned}
$$

The representation $\Lambda_{27}^{3}$ is isomorphic to the representation of $G_{2}$ in the space $S_{0}^{2}\left(\mathbb{R}^{7}\right)$ of all traceless symmetric bilinear forms.

Proposition 4.2. The map $\Phi: \mathbb{R}^{7} \otimes \mathfrak{g}_{2} \rightarrow \mathbb{R}^{7} \otimes S^{2}\left(\mathbb{R}^{7}\right)$ is injective.

Corollary 4.3. Let $\left(M^{7}, g, \omega^{3}\right)$ be an oriented, 7-dimensional Riemannian manifold with a fixed $G_{2}$-structure $\omega^{3}$. Then there exists at most one affine connection $\nabla$ such that $\nabla \omega^{3}=0$ and the torsion tensor $T$ is a 3 -form.

Proof. Given $\Sigma \in \mathbb{R}^{7} \otimes \mathfrak{g}_{2}$, the condition $\Phi(\Sigma)=0$ is equivalent to

$$
Z\lrcorner \Sigma(Y)+Y\lrcorner \Sigma(Z)=0
$$

for any two vectors $Y, Z \in \mathbb{R}^{7}$. Using the standard basis $e_{\alpha} \wedge e_{\beta}$ of the Lie algebra $\mathfrak{s o}(7)$ we decompose the elements $\Sigma\left(e_{i}\right) \in \mathfrak{g}_{2} \subset \mathfrak{s o}(7)$,

$$
\Sigma\left(e_{i}\right):=\sum_{1 \leq \alpha, \beta \leq 7} \omega_{i \alpha \beta} \cdot e_{\alpha} \wedge e_{\beta} .
$$


The condition $\Phi(\Sigma)=0$ implies $\omega_{i \alpha \beta}=-\omega_{\alpha i \beta}$. Consequently, $\Sigma$ depends on 35 parameters $\omega_{i \alpha \beta}(1 \leq i<\alpha<\beta \leq 7)$. Moreover, $\Sigma\left(e_{i}\right)$ belongs to the Lie-Algebra $\mathfrak{g}_{2}$ and hence we obtain seven equations for any index $i=1,2, \ldots, 7$. Altogether, these are 49 equations for 35 variables and a careful examination of the system yields the result that $\omega_{i \alpha \beta}=0$ is the only solution.

The low-dimensional $G_{2}$-representations, their highest weights, dimensions etc. are listed in the following table (see [22]):

\begin{tabular}{|c|c|c|}
\hline highest weight & dimension & space \\
\hline$(0,0)$ & 1 & $\Lambda_{1}^{0}:=\Lambda^{0}\left(\mathbb{R}^{7}\right)=\Lambda_{1}^{3}$ \\
$(1,0)$ & 7 & $\Lambda_{7}^{1}:=\Lambda^{1}\left(\mathbb{R}^{7}\right)=\Lambda_{7}^{2}=\Lambda_{7}^{3}$ \\
$(0,1)$ & 14 & $\mathfrak{g}_{2}=\Lambda_{14}^{2}$ \\
$(2,0)$ & 27 & $\Lambda_{27}^{3}=S_{0}^{2}\left(\mathbb{R}^{7}\right)$ \\
$(1,1)$ & 64 & $\Lambda_{64}$ \\
$(3,0)$ & 77 & $\Lambda_{77}$ \\
\hline
\end{tabular}

We now compute the $G_{2}$-decomposition of the three representations related with the maps $\Psi$ and $\Phi$.

Proposition 4.4. The following decompositions into irreducible $G_{2}$ representations hold:

1) $\mathbb{R}^{7} \otimes \mathfrak{m}=\Lambda_{1}^{0} \oplus \Lambda_{7}^{1} \oplus \Lambda_{14}^{2} \oplus \Lambda_{27}^{3} ;$

2) $\mathbb{R}^{7} \otimes \mathfrak{g}_{2}=\Lambda_{7}^{1} \oplus \Lambda_{27}^{3} \oplus \Lambda_{64}$

3) $\mathbb{R}^{7} \otimes S^{2}\left(\mathbb{R}^{7}\right)=2 \Lambda_{7}^{1} \oplus \Lambda_{14}^{2} \oplus \Lambda_{27}^{3} \oplus \Lambda_{64} \oplus \Lambda_{77}$.

Proof. The first decomposition is an elementary one, the second and third decomposition can be obtained using a suitable computer programme.

Since the $G_{2}$-map $\Phi: \mathbb{R}^{7} \otimes \mathfrak{g}_{2} \rightarrow \mathbb{R}^{7} \otimes S^{2}\left(\mathbb{R}^{7}\right)$ is injective and the multiplicity of $\Lambda_{27}^{3}$ in $\mathbb{R}^{7} \otimes S^{2}\left(\mathbb{R}^{7}\right)$ is one, we obtain

\section{COROLLARY 4.5 .}

1) $\Psi\left(\Lambda_{1}^{0} \oplus \Lambda_{27}^{3}\right) \subset \operatorname{Im}(\Phi)$

2) $\Psi\left(\Lambda_{14}^{2}\right) \cap \operatorname{Im}(\Phi)=0$.

Finally, we have to decide whether or not the space $\Psi\left(\Lambda_{7}^{1}\right)$ is contained in the image of $\Phi$. Since the representation $\Lambda_{7}^{1}$ has multiplicity two in $\mathbb{R}^{7} \otimes S^{2}\left(\mathbb{R}^{7}\right)$, we cannot use a universal argument is before. 
Proposition 4.6. $\Psi\left(\Lambda_{7}^{1}\right)$ is contained in $\operatorname{Im}(\Phi)$.

Proof. First of all we compute $\Psi(\Gamma)$ for a given vector $\Gamma \in \Lambda_{7}^{1}$. The element in $\mathbb{R}^{7} \otimes \mathbb{R}^{7}$ related to $\Gamma$ is the 2-form (skew-symmetric endomorphism) $\Gamma\lrcorner \omega^{3}$ and therefore we obtain

$$
\begin{aligned}
\Psi(\Gamma)(X, Y, Y) & =2 \sum_{i=1}^{7} \omega^{3}\left(\Gamma, Y, e_{i}\right) \cdot \omega^{3}\left(e_{i}, X, Y\right) \\
& =2 g(\Gamma, X) \cdot g(Y, Y)-2 g(\Gamma, X) \cdot g(X, Y)
\end{aligned}
$$

Suppose now that $\Psi\left(\Lambda_{7}^{1}\right) \subset \operatorname{Im}(\Phi)$. Since $\Phi$ is injective, there should exist a map $\Sigma: \Lambda_{7}^{1} \rightarrow \mathbb{R}^{7} \otimes \mathfrak{g}_{2}$ such that

$$
\Phi(\Sigma(\Gamma))=\Psi(\Gamma)
$$

The multiplicity of $\Lambda_{7}^{1}$ in $\mathbb{R}^{7} \otimes \mathfrak{g}_{2}$ equals one. Consequently, $\Sigma(\Gamma)$ is proportional to the map

$$
\Sigma_{0}(\Gamma)(Y):=\operatorname{pr}_{\mathfrak{g}_{2}}(\Gamma \wedge Y)
$$

where $\operatorname{pr}_{\mathfrak{g}_{2}}: \Lambda^{2}\left(\mathbb{R}^{7}\right)=\mathfrak{s o}(7) \rightarrow \mathfrak{g}_{2}$ is the orthogonal projection. The 2 -forms

$$
\left.\left.\frac{1}{\sqrt{3}} e_{1}\right\lrcorner \omega^{3}, \quad \cdots \quad, \frac{1}{\sqrt{3}} e_{7}\right\lrcorner \omega^{3}
$$

constitute an orthonormal basis of the subspace $\mathfrak{m} \subset \mathfrak{s o}(7)$ and the projection is given by the formula

$$
\left.\left.\operatorname{pr}_{\mathfrak{g}_{2}}\left(\alpha^{2}\right)=\alpha^{2}-\frac{1}{3} \sum_{i=1}^{7}\left(e_{i}\right\lrcorner \omega^{3}, \alpha^{2}\right) \cdot\left(e_{i}\right\lrcorner \omega^{3}\right) .
$$

Now we compute $\Phi\left(\Sigma_{0}(\Gamma)\right)$ :

$$
\begin{aligned}
\Phi\left(\Sigma_{0}(\Gamma)\right)(X, Y, Y) & \left.=2 \cdot g(X\lrcorner \operatorname{pr}_{\mathfrak{g}_{2}}(\Gamma \wedge Y), Y\right) \\
& \left.=2 \cdot(\Gamma \wedge Y)(X, Y)-\frac{2}{3} \sum_{i=1}^{7}\left(e_{i}\right\lrcorner \omega^{3}, \Gamma \wedge Y\right) \cdot \omega^{3}\left(e_{i}, X, Y\right) .
\end{aligned}
$$

Finally, we obtain

$$
\Phi\left(\Sigma_{0}(\Gamma)\right)(X, Y, Y)=\frac{2}{3} \Psi(\Gamma)(X, Y, Y)
$$

and the latter formula proves the Proposition.

To summarize the previous discussion we proved the following theorem.

Theorem 4.7. Let $\left(M^{7}, g, \omega^{3}\right)$ be a 7-dimensional Riemannian manifold with a $G_{2}$-structure $\omega^{3}$. The following conditions are equivalent:

1) The $\Lambda_{14}^{2}$-component of $\Gamma$ is zero. 
2) There exists an affine connection $\nabla$ with totally skew-symmetric torsion preserving the $G_{2}$-structure,

$$
\nabla \omega^{3}=0
$$

In this case the connection $\nabla$ is unique.

The Riemannian covariant derivative $\nabla^{g} \omega^{3}$ of the $G_{2}$-structure is given by the formula

$$
\nabla^{g} \omega^{3}=\varrho_{3}(\Gamma)\left(\omega^{3}\right),
$$

where $\varrho_{3}$ is the differential of the representation of $S O(7)$ in $\Lambda^{3}\left(\mathbb{R}^{7}\right)$. The $G_{2}$-map of $\mathbb{R}^{7} \otimes \mathfrak{m}$ into $\mathbb{R}^{7} \otimes \Lambda^{3}\left(\mathbb{R}^{7}\right)$ given by the formula $X \otimes Y \rightarrow X \otimes \varrho_{3}(Y) \omega^{3}$ is injective (see [15]). Consequently, the different geometric classes of $G_{2}$-structures introduced by Fernandez/Gray (see [11]) using the covariant derivative $\nabla^{g} \omega^{3}$ can be defined via the algebraic type of $\Gamma \in \Lambda^{1}\left(M^{7}\right) \otimes \Lambda^{1}\left(M^{7}\right)$. The condition $\Gamma \in \Lambda_{1}^{0} \oplus \Lambda_{7}^{1} \oplus \Lambda_{27}^{3}$ characterizes the so-called integrable $G_{2}$-structures, i.e., the $G_{2}$-structures of type $W_{1} \oplus W_{4} \oplus W_{3}$ in the notation of [11]. Let us decompose $\Gamma$ into its three parts:

$$
\Gamma=\lambda \cdot \operatorname{Id}_{T\left(M^{7}\right)} \oplus \beta \oplus \Gamma_{27}^{3} .
$$

The $\Lambda_{1}^{0}$-part acts as a map $\lambda \cdot \operatorname{Id}_{T\left(M^{7}\right)}: \mathbb{R}^{7} \rightarrow \mathfrak{m}$ via the formula

$$
\left.\left(\lambda \cdot \operatorname{Id}_{T\left(M^{7}\right)}\right)(X):=\frac{\lambda}{12} \cdot(X\lrcorner \omega^{3}\right) .
$$

The component $\beta \in \Lambda_{7}^{1}$ is a vector field and we will use the embedding $\mathbb{R}^{7} \subset \mathbb{R}^{7} \otimes \mathfrak{m}$ given by the equation

$$
\left.\left.\beta(X):=\frac{1}{4} \cdot \operatorname{pr}_{\mathfrak{m}}(\beta \wedge X)=\frac{1}{12} \sum_{i=1}^{7}\left(\beta \wedge X, e_{i}\right\lrcorner \omega^{3}\right) \cdot\left(e_{i}\right\lrcorner \omega^{3}\right) .
$$

The third component $\Gamma_{27}^{3} \in \Lambda_{27}^{3}$ is a 3 -form defining a map $\Gamma_{27}^{3}: \mathbb{R}^{7} \rightarrow \mathfrak{m}$ by the formula

$$
\left.\left.\left.\left.\Gamma_{27}^{3}(X):=\frac{1}{2} \cdot \operatorname{pr}_{\mathfrak{m}}(X\lrcorner \Gamma_{27}^{3}\right)=\frac{1}{6} \sum_{i=1}^{7}(X\lrcorner \Gamma_{27}^{3}, e_{i}\right\lrcorner \omega^{3}\right) \cdot\left(e_{i}\right\lrcorner \omega^{3}\right) .
$$

We describe the action $\varrho_{3}\left(\alpha^{2}\right)$ of a 2 -form $\alpha^{2} \in \Lambda^{2}\left(\mathbb{R}^{7}\right)=\mathfrak{s o}(7)$ on the form $\omega^{3}$. Suppose that the projection of $\alpha^{2}$ onto the space $\Lambda_{7}^{2}$ is given by a vector $Z \in \mathbb{R}^{7}$,

$$
\left.\operatorname{pr}_{\mathfrak{m}}\left(\alpha^{2}\right)=Z\right\lrcorner \omega^{3} .
$$

Then the representation $\varrho_{3}\left(\alpha^{2}\right)$ acts on the 3 -form $\omega^{3}$ by the formula (see [19])

$$
\left.\varrho_{3}\left(\alpha^{2}\right)\left(\omega^{3}\right)=-3 \cdot(Z\lrcorner * \omega^{3}\right) .
$$

Consequently, we obtain a formula for the covariant derivative $\nabla^{g} \omega^{3}$ involving the function $\lambda$, the vector field $\beta$ as well as the 3 -form $\Gamma_{27}^{3}$ :

$$
\left.\left.\left.\left.\nabla_{X}^{g} \omega^{3}=-\frac{\lambda}{4} \cdot(X\lrcorner * \omega^{3}\right)-\sum_{i=1}^{7}\left(\frac{1}{4} \beta \wedge X+\frac{1}{2} X\right\lrcorner \Gamma_{27}^{3}, e_{i}\right\lrcorner \omega^{3}\right) \cdot\left(e_{i}\right\lrcorner * \omega^{3}\right) .
$$


Now we can express the differential $d \omega^{3}$ and the codifferential $\delta^{g}\left(\omega^{3}\right)$ of the 3 -form by $\Gamma$. For example, we have

$$
\left.\left.\left.\left.\left.\delta^{g}\left(\omega^{3}\right)=-\sum_{j=1}^{7} e_{j}\right\lrcorner \nabla_{e_{j}}^{g} \omega^{3}=\sum_{i, j=1}^{7}\left(\frac{1}{4} \beta \wedge e_{j}+\frac{1}{2} e_{j}\right\lrcorner \Gamma_{27}^{3}, e_{i}\right\lrcorner \omega^{3}\right) \cdot\left(e_{j}\right\lrcorner\left(e_{i}\right\lrcorner * \omega^{3}\right)\right) .
$$

The map

$$
\left.\left.\left.\left.\Gamma_{27}^{3} \in \Lambda_{27}^{3} \longmapsto \sum_{i, j=1}^{7}\left(e_{j}\right\lrcorner \Gamma_{27}^{3}, e_{i}\right\lrcorner \omega^{3}\right) \cdot\left(e_{j}\right\lrcorner\left(e_{i}\right\lrcorner * \omega^{3}\right)\right) \in \Lambda^{2}\left(\mathbb{R}^{7}\right)=\Lambda_{7}^{2} \oplus \Lambda_{14}^{2}
$$

is obviously trivial and the map

$$
\left.\left.\left.\beta \in \mathbb{R}^{7} \longmapsto \sum_{i, j=1}^{7}\left(\beta \wedge e_{j}, e_{i}\right\lrcorner \omega^{3}\right) \cdot\left(e_{j}\right\lrcorner\left(e_{i}\right\lrcorner * \omega^{3}\right)\right) \in \Lambda^{2}\left(\mathbb{R}^{7}\right)=\Lambda_{7}^{2} \oplus \Lambda_{14}^{2}
$$

must be proportional to the map $\beta \rightarrow \beta\lrcorner \omega^{3}$ since the multiplicity of the 7-dimensional component in $\Lambda^{2}\left(\mathbb{R}^{7}\right)$ is one. Computing the constant we obtain

$$
\left.\left.\left.\left.\sum_{i, j=1}^{7}\left(\beta \wedge e_{j}, e_{i}\right\lrcorner \omega^{3}\right) \cdot\left(e_{j}\right\lrcorner\left(e_{i}\right\lrcorner * \omega^{3}\right)\right)=-4 \cdot(\beta\lrcorner \omega^{3}\right)
$$

and, finally,

$$
\left.\delta^{g}\left(\omega^{3}\right)=-\beta\right\lrcorner \omega^{3} .
$$

We handle the differential $d \omega^{3}$ in a similar way. Using again that the multiplicities of $\mathbb{R}^{7}$ and $\Lambda_{27}^{3}$ in the $G_{2}$-representation $\Lambda^{4}\left(\mathbb{R}^{7}\right)$ are one we verify the idendities

$$
\begin{aligned}
& \left.\left.\left.\sum_{i, j=1}^{7}\left(e_{j}\right\lrcorner \Gamma_{27}^{3}, e_{i}\right\lrcorner \omega^{3}\right) \cdot\left(e_{j} \wedge\left(e_{i}\right\lrcorner * \omega^{3}\right)\right)=-2 \cdot\left(* \Gamma_{27}^{3}\right), \\
& \left.\left.\sum_{i, j=1}^{7}\left(\beta \wedge e_{j}, e_{i}\right\lrcorner \omega^{3}\right) \cdot\left(e_{j} \wedge\left(e_{i}\right\lrcorner * \omega^{3}\right)\right)=-3 \cdot\left(\beta \wedge \omega^{3}\right) .
\end{aligned}
$$

Then we obtain the formula

$$
d \omega^{3}=\sum_{i=1}^{7} e_{i} \wedge \nabla_{e_{i}}^{g} \omega^{3}=-\lambda \cdot\left(* \omega^{3}\right)+* \Gamma_{27}^{3}+\frac{3}{4} \cdot\left(\beta \wedge \omega^{3}\right)
$$

We compute now the 1-form $\Sigma(\Gamma) \in \mathbb{R}^{7} \otimes \mathfrak{g}_{2}$ defined by the condition $\Phi(\Sigma(\Gamma))=\Psi(\Gamma)$. Since $\Psi(\lambda \cdot \mathrm{Id})=0$ we have $\Sigma(\lambda \cdot \mathrm{Id})=0$, i.e., $\Sigma(\Gamma)$ does not depend on the $\Lambda_{1}^{0}$-part of $\Gamma$. The 1 -form $\Psi(\Gamma)$ with values in $S^{2}\left(\mathbb{R}^{7}\right)$ is given by

$$
\left.\left.\Psi(\Gamma)(X, Y, Y)=2 \cdot g(\Gamma(Y)(X), Y)=g(X\lrcorner \operatorname{pr}_{\mathfrak{m}}(Y\lrcorner \Gamma_{27}^{3}+\frac{1}{2} \beta \wedge Y\right), Y\right) .
$$

On the other hand, let us introduce the map $\Sigma: \Lambda_{7}^{1} \oplus \Lambda_{27}^{3} \rightarrow \mathbb{R}^{7} \otimes \mathfrak{g}_{2}$ given by the formula

$$
\left.\Sigma(\Gamma)(Y):=-\frac{1}{2} \cdot \operatorname{pr}_{\mathfrak{g}_{2}}(Y\lrcorner \Gamma_{27}^{3}-\frac{1}{4} \beta \wedge Y\right)
$$


Proposition 4.6 and a direct calculation yields that $\Sigma(\Gamma)$ is indeed the 1-form with values in the Lie algebra $\mathfrak{g}_{2}$ corresponding to $\Gamma$ :

$$
\begin{aligned}
\Phi\left(\Sigma\left(\Gamma_{27}^{3}\right)\right)(X, Y, Y) & \left.\left.=-g(X\lrcorner \operatorname{pr}_{\mathfrak{g}_{2}}(Y\lrcorner \Gamma_{27}^{3}\right), Y\right) \\
& \left.\left.\left.=-(Y\lrcorner \Gamma_{27}^{3}\right)(X, Y)+g(X\lrcorner \operatorname{pr}_{\mathfrak{m}}(Y\lrcorner \Gamma_{27}^{3}\right), Y\right) \\
& =\Psi\left(\Gamma_{27}^{3}\right)(X, Y, Y) .
\end{aligned}
$$

We introduce a new 1-form $\Gamma^{*}(X):=\Gamma(X)-\Sigma(\Gamma(X))$,

$$
\left.\left.\Gamma^{*}(X)=\frac{\lambda}{12} \cdot(X\lrcorner \omega^{3}\right)+\frac{1}{2} \cdot(X\lrcorner \Gamma_{27}^{3}\right)+\frac{3}{8} \cdot \operatorname{pr}_{\mathfrak{m}}(\beta \wedge X)-\frac{1}{8} \cdot(\beta \wedge X)
$$

and remark that the torsion form $T$ is given by

$$
T(X, Y, Z)=-g\left(\Gamma^{*}(X)(Y), Z\right)+g\left(\Gamma^{*}(Y)(X), Z\right) .
$$

Consequently, we obtain a formula for the torsion form $T$,

$$
T=-\frac{\lambda}{6} \cdot \omega^{3}-\Gamma_{27}^{3}+T_{\beta},
$$

where the 3 -form $T_{\beta}$ is defined by the equation

$$
\begin{aligned}
T_{\beta}(X, Y, Z):= & \frac{3}{8} \cdot\left(\operatorname{pr}_{\mathfrak{m}}(\beta \wedge Y)(X, Z)-\operatorname{pr}_{\mathfrak{m}}(\beta \wedge X)(Y, Z)\right) \\
& +\frac{1}{8} \cdot(g(\beta, Y) \cdot g(X, Z)-g(\beta, X) \cdot g(Y, Z)) .
\end{aligned}
$$

The map $\beta \in \mathbb{R}^{7} \longmapsto T_{\beta} \in \Lambda^{3}\left(\mathbb{R}^{7}\right)$ must be a multiple of the map $\left.\beta \longmapsto \beta\right\lrcorner * \omega^{3}$ since the multiplicity of $\mathbb{R}^{7}$ in $\Lambda^{3}\left(\mathbb{R}^{7}\right)$ is one. Computing algebraically the constant we obtain the equation

$$
\left.T_{\beta}=-\frac{1}{4} \cdot(\beta\lrcorner * \omega^{3}\right) .
$$

We thus computed the torsion form of the unique connection preserving the $G_{2^{-}}$ structure:

$$
\left.T=-\frac{\lambda}{6} \cdot \omega^{3}-\Gamma_{27}^{3}-\frac{1}{4} \cdot(\beta\lrcorner * \omega^{3}\right) .
$$

Using the equations for the exterior differential and the codifferential of the form $\omega^{3}$ we can substitute the function $\lambda$ as well as the 3 -form $\Gamma_{27}^{3}$ :

$$
\lambda=-\frac{1}{7} \cdot\left(d \omega^{3}, * \omega^{3}\right), \quad \Gamma_{27}^{3}=* d \omega^{3}+\lambda \cdot \omega^{3}-\frac{3}{4} *\left(\beta \wedge \omega^{3}\right) .
$$

For any vector $\beta$ we have

$$
\left.*\left(\beta \wedge \omega^{3}\right)=-(\beta\lrcorner * \omega^{3}\right) .
$$

Let us summarize the result. 
THEOREM 4.8. Let $\left(M^{7}, g, \omega^{3}\right)$ be a 7-dimensional $G_{2}$-manifold of type $\Lambda_{1}^{0} \oplus \Lambda_{7}^{1} \oplus$ $\Lambda_{27}^{3}$. The torsion form of the unique affine connection $\nabla$ preserving the structure with totally skew-symmetric torsion is given by the formula

$$
T=\frac{1}{6} \cdot\left(d \omega^{3}, * \omega^{3}\right) \cdot \omega^{3}-* d \omega^{3}+*\left(\beta \wedge \omega^{3}\right) .
$$

The vector field $\beta$ as well as the differential and the codifferential of the 3-form $\omega^{3}$ are related by

$$
\left.\delta^{g}\left(\omega^{3}\right)=-(\beta\lrcorner \omega^{3}\right), \quad d \omega^{3}=\frac{1}{7} \cdot\left(d \omega^{3}, * \omega^{3}\right) \cdot\left(* \omega^{3}\right)+* \Gamma_{27}^{3}+\frac{3}{4} \cdot\left(\beta \wedge \omega^{3}\right) .
$$

The particular case $\Gamma=\lambda \cdot \operatorname{Id}_{T\left(M^{7}\right)}$ corresponds to nearly parallel $G_{2}$-structures. In this case, the Riemannian manifold $\left(M^{7}, g\right)$ is Einstein, the parameter $\lambda$ is constant and related to the scalar curvature of $M^{7}$ (see [19]).

COROLlary 4.9. Let $\left(M^{7}, g, \omega^{3}\right)$ be a 7-dimensional Riemannian manifold with a nearly parallel $G_{2}$-structure $(\Gamma=\lambda$. Id). Then there exists a unique affine connection $\nabla$ such that

$$
\nabla \omega^{3}=0 \text { and } T \text { is a 3-form. }
$$

The torsion tensor is given by the formula $6 \cdot T=\left(d \omega^{3}, * \omega^{3}\right) \cdot \omega^{3} . T$ is $\nabla$-parallel and coclosed, $\nabla T=\delta T=0$.

Corollary 4.10. Let $\left(M^{7}, g, \omega^{3}\right)$ be a 7-dimensional nearly parallel $G_{2}$ manifold. Then the triple $\left(M^{7}, g, T^{*}:=3 \cdot T\right)$ is a solution of the string equations with constant dilation:

$$
\operatorname{Ric}_{i j}^{g}-\frac{1}{4} T_{i m n}^{*} T_{j m n}^{*}=0, \quad \delta^{g}\left(T^{*}\right)=0 .
$$

A cocalibrated $G_{2}$-structure is defined by the condition that $\omega^{3}$ is coclosed, $\delta^{g}\left(\omega^{3}\right)=0$. Equivalently, $\Gamma$ depends only on a function $\lambda$ and on a 3 -form $\Gamma_{27}^{3}$ of type $\Lambda_{27}^{3}$,

$$
\Gamma=\lambda \cdot \operatorname{Id}+\Gamma_{27}^{3} \text {. }
$$

The differential as well as the torsion form $T$ of cocalibrated $G_{2}$-structures are given by the formulas:

$$
\begin{aligned}
& d \omega^{3}=-\lambda \cdot\left(* \omega^{3}\right)+\left(* \Gamma_{27}^{3}\right), \quad \lambda=-\frac{1}{7} \cdot\left(d \omega^{3}, * \omega^{3}\right), \\
& T=-\left(* d \omega^{3}\right)-\frac{7}{6} \cdot \lambda \cdot \omega^{3}, \quad d * T=-\frac{7}{6} \cdot d \lambda \wedge * \omega^{3} .
\end{aligned}
$$

In particular, $T$ is coclosed if and only if $\lambda$ is constant. This occurs, for example, if the $G_{2}$-structure is of pure type $\Lambda_{1}^{0}$ or $\Lambda_{27}^{3}$.

Another distinguished class is $\Lambda_{7}^{1} \oplus \Lambda_{27}^{3}$. The torsion is given by

$$
T=-* d \omega^{3}+*\left(\beta \wedge \omega^{3}\right) \text {. }
$$


Examples of this type were presented and discussed in connection with type IIB supergravity solutions in [26] where this expression with a non trivial $\beta$ was first given, though by a different approach, making use of both Killing spinor equations. ${ }^{1}$.

The last class of $G_{2}$-structures we want to emphasize is $\Lambda_{1}^{0} \oplus \Lambda_{7}^{1}$. Then $\beta$ is a closed 1 -form and we obtain

$$
\begin{gathered}
\left.\delta^{g}\left(\omega^{3}\right)=-\beta\right\lrcorner \omega^{3}, \quad d \omega^{3}=-\lambda \cdot\left(* \omega^{3}\right)+\frac{3}{4} \cdot\left(\beta \wedge \omega^{3}\right), \\
T=-\frac{\lambda}{6} \cdot \omega^{3}+\frac{1}{4} *\left(\beta \wedge \omega^{3}\right)=-\frac{7 \lambda}{6} \cdot \omega^{3}-* d \omega^{3}+*\left(\beta \wedge \omega^{3}\right) .
\end{gathered}
$$

In particular, if $\lambda=0$, then the torsion form is coclosed, $\delta^{g}(T)=0$.

5. The $\nabla$-Ricci tensor of a $G_{2}$-structure. We consider a $G_{2}$-manifold $\left(M^{7}, g, \omega^{3}, \nabla\right)$ of type $\Lambda_{1}^{0} \oplus \Lambda_{7}^{1} \oplus \Lambda_{27}^{3}$ and its unique connection $\nabla$ preserving the $G_{2}$-structure. The totally skew-symmetric torsion tensor $T$ is a 3-form of type $\Lambda_{1}^{3} \oplus \Lambda_{7}^{3} \oplus \Lambda_{27}^{3}$. For any vector field $X$ the covariant derivative $\nabla_{X} T$ is again a 3-form of type $\Lambda_{1}^{3} \oplus \Lambda_{7}^{3} \oplus \Lambda_{27}^{3}$. Moreover, the $\nabla$-parallel 3-form $\omega^{3}$ defines a $\nabla$-parallel spinor field $\Psi_{0}$ (see [19]). The Clifford products $\left(\nabla_{X} T\right) \cdot \Psi_{0}$ and $\left.(X\lrcorner d T\right) \cdot \Psi_{0}$ depend only on the $\left(\Lambda_{1}^{3} \oplus \Lambda_{7}^{3}\right)$-part of the corresponding 3 -forms:

$$
\begin{aligned}
& \left.\left.\pi_{1}^{3}\left(\nabla_{X} T\right):=\frac{1}{7} \cdot\left(\nabla_{X} T, \omega^{3}\right) \cdot \omega^{3}, \quad \pi_{7}^{3}\left(\nabla_{X} T\right):=\frac{1}{4} \sum_{i=1}^{7}\left(\nabla_{X} T, e_{i}\right\lrcorner * \omega^{3}\right) \cdot\left(e_{i}\right\lrcorner * \omega^{3}\right), \\
& \left.\left.\left.\left.\left.\left.\pi_{1}^{3}(X\lrcorner d T\right):=\frac{1}{7} \cdot(X\lrcorner d T, \omega^{3}\right) \cdot \omega^{3}, \quad \pi_{7}^{3}(X\lrcorner d T\right):=\frac{1}{4} \sum_{i=1}^{7}(X\lrcorner d T, e_{i}\right\lrcorner * \omega^{3}\right) \cdot\left(e_{i}\right\lrcorner * \omega^{3}\right) .
\end{aligned}
$$

The second equation of Corollary 3.2 becomes

$$
\left.\frac{1}{2}(X\lrcorner d T\right) \cdot \Psi_{0}+\left(\nabla_{X} T\right) \cdot \Psi_{0}-\operatorname{Ric}^{\nabla}(X) \cdot \Psi_{0}=0 .
$$

Using the algebraic formulas

$$
\left.(X\lrcorner * \omega^{3}\right) \cdot \Psi_{0}=4 \cdot X \cdot \Psi_{0}, \quad \omega^{3} \cdot \Psi_{0}=-7 \cdot \Psi_{0}
$$

valid for the special spinor $\Psi_{0}$ related to $\omega^{3}$ we conclude

$$
\left.\left.\left.(X\lrcorner d T, \omega^{3}\right)=-2 \cdot\left(\nabla_{X} T, \omega^{3}\right), \quad \operatorname{Ric}^{\nabla}(X)=\frac{1}{2} \sum_{i=1}^{7}(X\lrcorner d T+2 \cdot \nabla_{X} T, e_{i}\right\lrcorner * \omega^{3}\right) \cdot e_{i} .
$$

The relation between the Ricci tensors

$$
\operatorname{Ric}^{\nabla}(X)=\operatorname{Ric}^{g}(X)+\frac{1}{4} \sum_{i, j=1}^{n} g\left(T\left(e_{i}, X\right), T\left(e_{j}, e_{i}\right)\right) \cdot e_{j}-\frac{1}{2} \sum_{i=1}^{n} \delta^{g}(T)\left(X, e_{i}\right) \cdot e_{i}
$$

\footnotetext{
${ }^{1}$ We thank J. Gauntlett for drawing our attention to their work, in particular to the possible class $\Lambda_{7}^{1}$ which was missing in a preliminary version of our paper.
} 
allows us to compute the Riemannian Ricci tensor. We summarize all the derived results in one theorem.

THEOREM 5.1. Let $\left(M^{7}, g, \omega^{3}, \nabla\right)$ be a $G_{2}$-manifold of type $\Lambda_{1}^{0} \oplus \Lambda_{7}^{1} \oplus \Lambda_{27}^{3}$ and its unique connection $\nabla$ preserving the $G_{2}$-structure. The Ricci tensor Ric ${ }^{\nabla}$ is given by the formula

$$
\left.\left.\operatorname{Ric}^{\nabla}(X)=\frac{1}{2} \sum_{i=1}^{7}(X\lrcorner d T+2 \cdot \nabla_{X} T, e_{i}\right\lrcorner * \omega^{3}\right) \cdot e_{i} .
$$

$T$ is a solution of the equation

$$
\left.\left.\operatorname{Ric}_{i j}^{g}-\frac{1}{4} T_{i m n} T_{j m n}-\frac{1}{2}\left(e_{i}\right\lrcorner d T+2 \cdot \nabla_{e_{i}} T, e_{j}\right\lrcorner * \omega^{3}\right)-\frac{1}{2} \delta^{g}(T)_{i j}=0,
$$

and satisfies, for any vector $X$, the condition $\left.(X\lrcorner d T, \omega^{3}\right)=-2 \cdot\left(\nabla_{X} T, \omega^{3}\right)$. There exists a $\nabla$-parallel spinor field $\Psi_{0}$ such that the 3-form $\omega^{3}$ and the Riemannian Dirac operator $D^{g}$ act on it by

$\left.\omega^{3} \cdot \Psi_{0}=-7 \cdot \Psi_{0}, \quad D^{g}\left(\Psi_{0}\right)=-\frac{3}{4} \cdot T \cdot \Psi_{0}=-\frac{7}{8} \cdot \lambda \cdot \Psi_{0}+\frac{3}{16} \cdot(\beta\lrcorner * \omega^{3}\right) \cdot \Psi_{0}$.

EXAMPLE 5.2. Consider the case of a nearly-parallel $G_{2}$-structure $\left(\Gamma=\lambda \cdot \operatorname{Id}_{T\left(M^{7}\right)}\right)$. The torsion form is proportional to the form of the $G_{2}$-structure,

$$
T=-\frac{\lambda}{6} \cdot \omega^{3}, \quad d \omega^{3}=-\lambda \cdot\left(* \omega^{3}\right), \quad d T=\frac{\lambda^{2}}{6} \cdot\left(* \omega^{3}\right) .
$$

Then we have

$\left.\left.\operatorname{Ric}_{i j}^{g}=\frac{27}{72} \cdot \lambda^{2} \cdot \delta_{i j}, \quad \frac{1}{4} T_{i m n} T_{j m n}=\frac{3}{72} \cdot \lambda^{2} \cdot \delta_{i j}, \quad \frac{1}{2}\left(e_{i}\right\lrcorner d T, e_{j}\right\lrcorner * \omega^{3}\right)=\frac{24}{72} \cdot \lambda^{2} \cdot \delta_{i j}$.

The formula of Theorem 5.1 for the Ricci tensor generalizes the well-known fact that a nearly-parallel $G_{2}$-manifold is an Einstein space (see [19]).

We consider now only the cocalibrated case, $\beta=0$. Then the covariant derivative $\nabla_{X} T$ is a 3 -form of type $\Lambda_{1}^{3} \oplus \Lambda_{27}^{3}$ and the formula for the Ricci tensor does not contain the $\nabla_{X} T$-term. If $M^{7}$ is a compact, cocalibrated $G_{2}$-manifold we can apply the estimate for the first eigenvalue of the Riemannian Dirac operator (see [13])

$$
\frac{7}{4 \cdot 6}\left\|\Psi_{0}\right\|^{2} \cdot \operatorname{vol}\left(M^{7}\right) \cdot \operatorname{Scal}_{m i n}^{g} \leq \int_{M^{7}}\left(D^{g} \Psi_{0}, D^{g} \Psi_{0}\right)
$$

where $\mathrm{Scal}_{\min }^{g}$ denotes the minimum of the Riemannian scalar curvature. Using the equation $8 \cdot D^{g}\left(\Psi_{0}\right)=-7 \cdot \lambda \cdot \Psi_{0}$ and the definition of the function $\lambda$ we obtain an $L^{2}$-lower bound for $\left(d \omega^{3}, * \omega^{3}\right)$.

TheOREM 5.3. For any compact, cocalibrated $G_{2}$-manifold the following inequality holds

$$
\frac{56}{3} \cdot \operatorname{vol}\left(M^{7}\right) \cdot \mathrm{Scal}_{\min }^{g} \leq \int_{M^{7}}\left(d \omega^{3}, * \omega^{3}\right)^{2}
$$


Equality occurs if and only if the cocalibrated $G_{2}$-structure is nearly parallel. If the $G_{2}$ structure is of pure type $\Lambda_{27}^{3}$, then the minimum of the scalar curvature is non-positive.

The formula of Theorem 5.1 computes in particular the Ricci tensor and the scalar curvature of the connection $\nabla$ :

$$
\left.\left.\left.\left.\operatorname{Ric}_{i j}^{\nabla}=\frac{1}{2}\left(e_{i}\right\lrcorner d T, e_{j}\right\lrcorner * \omega^{3}\right), \quad \operatorname{Scal}^{\nabla}=\frac{1}{2} \sum_{i=1}^{7}\left(e_{i}\right\lrcorner d T, e_{i}\right\lrcorner * \omega^{3}\right) .
$$

A cocalibrated $G_{2}$-structure $\left(M^{\top}, \omega^{3}, \nabla, \Psi_{0}\right)$ together with its canonical connection and spinor field solves all of the three string equations $\operatorname{Ric}^{\nabla}=0, \nabla \Psi_{0}=0, T \cdot \Psi_{0}=0$ if and only if the $G_{2}$-structure is geometrically flat $(\lambda=0=T)$. Therefore we study the first of these equations only.

Theorem 5.4. Let $\left(M^{7}, g, \omega^{3}\right)$ be a 7-dimensional Riemannian manifold with a cocalibrated $G_{2}$-structure. The following conditions are equivalent:

1) The Ricci tensor $\operatorname{Ric}^{\nabla}$ vanishes.

2). The torsion form $T$ is closed and coclosed, $d T=0=\delta^{g}(T)$.

3) $\lambda$ is constant and the $G_{2}$-structure $\omega^{3}$ satisfies the equation

$$
d * d \omega^{3}+\frac{7}{6} \cdot \lambda \cdot d \omega^{3}=0 \quad \lambda=-\frac{1}{7} \cdot\left(d \omega^{3}, * \omega^{3}\right) .
$$

Moreover, in this case we have

$$
\left(* d \omega^{3}+\frac{7}{6} \cdot \lambda \cdot \omega^{3}\right) \wedge d \omega^{3}=0 .
$$

If the $G_{2}$-structure is of pure type $\Lambda_{1}^{0}$ or $\Lambda_{27}^{3}$ and $\operatorname{Ric}^{\nabla}$ vanishes, then the Riemannian manifold $M^{7}$ is a Ricci flat space with holonomy $G_{2}$.

Proof. The condition $\operatorname{Ric}^{\nabla}=0$ means that $\left.X\right\lrcorner d T$ is orthogonal to the subspace $\left.\{Y\lrcorner * \omega^{3}\right\}=\Lambda_{7}^{1}$. Moreover, $\left.X\right\lrcorner d T$ is orthogonal to $\omega^{3}$ and therefore it belongs to the subspace $\Lambda_{27}^{3}$. Consequently we obtain for any vector $X$ the conditions

$$
\left.(X\lrcorner d T) \wedge \omega^{3}=0 \quad \text { and } \quad(X\lrcorner d T\right) \wedge * \omega^{3}=0 .
$$

The subspace of all 4-forms satisfying these algebraic equations is a $G_{2}$-invariant subspace of $\Lambda^{4}$ and it is not hard to see that this space is trivial, i.e. we conclude that $d T=0$. Since $\delta^{g}(T)$ is the antisymmetric part of the Ricci tensor Ric ${ }^{\nabla}$, the codifferential of the torsion form must vanish, too. These arguments prove the equivalence of the three conditions in the Theorem. The torsion form is of type $\Lambda_{1}^{0} \oplus \Lambda_{27}^{3}$ and we differentiate the equation $T \wedge \omega^{3}=0$. Then we obtain the last equation of the Theorem.

REMARK 5.5. The cocalibrated $G_{2}$-structure $\left(M^{7}, g, \omega^{3}, \nabla\right)$ defines a (homogeneous) solution to the string equations with constant dilation

$$
\operatorname{Ric}_{i j}^{g}-\frac{1}{4} T_{i m n} T_{j m n}=0, \quad \delta^{g}(T)=0
$$

if and only if $\omega^{3}$ is a solution of the cubic equation

$$
d * d \omega^{3}+\frac{7}{6} \cdot \lambda \cdot d \omega^{3}=0
$$


and $7 \cdot \lambda=-\left(d \omega^{3}, * \omega^{3}\right)$ is constant. In this case the torsion form is closed and coclosed. In particular, if $M^{7}$ is a non-flat $G_{2}$-structure, we obtain a necessary topological condition:

$$
H^{3}\left(M^{7} ; \mathbb{Z}\right) \neq 0
$$

We have not succeeded in constructing any cocalibrated $G_{2}$-structure satisfying this non-linear equation.

THEOREM 5.6. Let $\left(M^{7}, g, \omega^{3}, \nabla\right)$ be a 7-dimensional compact nearly parallel $G_{2}$-manifold and $\nabla$ be the unique $G_{2}$-connection with totally skew-symmetric torsion. Then every $\nabla$-harmonic spinor $\Psi$ is $\nabla$-parallel. Moreover, the space of $\nabla$-parallel spinors is one-dimensional.

Proof. The Dirac operator $D$ is selfadjoint. Let $M^{7}$ be compact and consider a $\nabla$-harmonic spinor, $D \Psi=0$. Then Theorem 3.3 implies

$$
\left.\sum_{i=1}^{7} \int_{M^{7}}\left(e_{i}\right\lrcorner T \cdot \nabla_{e_{i}} \Psi, \Psi\right)=0,
$$

since $d T=2 \cdot \sigma^{T}$ holds in case of a nearly-parallel structure. Using the latter equality as well as the Schrödinger-Lichnerowicz formula we obtain

$$
\int_{M^{7}}\left(\|\nabla \Psi\|^{2}+\frac{1}{2}(d T \cdot \Psi, \Psi)+\frac{1}{4} \operatorname{Scal}^{\nabla}\|\Psi\|^{2}\right)=0 .
$$

The 4-form $* \omega^{3}$ acts on spinors as a symmetric endomorphism with the eigenvalues +1 and -7 . The result follows now from the estimate

$$
\frac{1}{2}(d T \cdot \Psi, \Psi)+\frac{1}{4} \operatorname{Scal}^{\nabla}\|\Psi\|^{2} \geq\left(\frac{1}{2} \cdot 24 \cdot \lambda^{2}(-7)+\frac{1}{4} \cdot 48 \cdot \lambda^{2} \cdot 7\right)\|\Psi\|^{2}=0 .
$$

REMARK 5.7. Let us discuss the result from the point of view of the spectrum of the Riemannian Dirac operator. The first eigenvalue of the Riemannian Dirac operator on a compact, simply connected nearly parallel manifold $\left(M^{7}, g, \omega^{3}, \nabla\right)$ is

$$
\mu=\frac{1}{2} \sqrt{\frac{7 \cdot R}{6}}=\frac{7 \cdot \lambda}{8}
$$

(see [13]). Here we have constructed a $\nabla$-parallel spinor

$$
\left.\nabla \Psi_{0}=\nabla_{X}^{g} \Psi_{0}-\frac{1}{24} \cdot \lambda \cdot(X\lrcorner \omega^{3}\right) \cdot \Psi_{0}=0 .
$$

Let us compute the Riemannian Dirac operator :

$$
D^{g} \Psi_{0}-\frac{3}{24} \cdot \lambda \cdot \omega^{3} \cdot \Psi_{0}=0 .
$$

The endomorphism $\omega^{3}$ acts $\Psi_{0}$ by multiplication by -7 . Therefore we obtain

$$
D^{g} \Psi_{0}=-\frac{7}{8} \cdot \lambda \cdot \Psi_{0}
$$

i.e. the $\nabla$-parallel spinor field on $M^{7}$ is the real Killing spinor on $M^{7}$. In this sense the $\nabla$-parallel spinors on (non-nearly parallel) cocalibrated $G_{2}$-structures generalize the Killing spinors. 
6. Examples. Denote by $\mathrm{H}(3)$ the 6-dimensional, simply-connected Heisenberg group and consider the product $M^{7}:=\mathrm{H}(3) \times \mathbb{R}$. There exists a left invariant metric and an orthonormal frame $e_{1}, \ldots, e_{7}$ such that the corresponding 3 -form $\omega^{3}$ defines a cocalibrated $G_{2}$-structure of pure type $\Lambda_{27}^{3}$ (see [12]). Indeed, the exterior differentials are given by the formulas

$$
\begin{gathered}
d e_{1}=d e_{2}=d e_{3}=d e_{6}=d e_{7}=0 \\
d e_{4}=e_{1} \wedge e_{6}+e_{3} \wedge e_{7}, \quad d e_{5}=e_{1} \wedge e_{3}-e_{6} \wedge e_{7}
\end{gathered}
$$

and an easy computation yields the following formula for the differential

$$
d \omega^{3}=e_{1} \wedge e_{2} \wedge e_{3} \wedge e_{4}+e_{2} \wedge e_{4} \wedge e_{6} \wedge e_{7}+e_{1} \wedge e_{2} \wedge e_{5} \wedge e_{6}-e_{2} \wedge e_{3} \wedge e_{5} \wedge e_{7}
$$

We see that $d \omega^{3} \wedge \omega^{3}=0, d \omega^{3} \wedge * \omega^{3}=0$, i.e., the $G_{2}$-structure is of pure type $\Lambda_{27}^{3}$. The torsion form $T=-* d \omega^{3}$ equals

$$
T=-\left(e_{5} \wedge e_{6} \wedge e_{7}-e_{1} \wedge e_{3} \wedge e_{5}+e_{3} \wedge e_{4} \wedge e_{7}+e_{1} \wedge e_{4} \wedge e_{6}\right)
$$

and its differential is given by

$$
d T=-4 \cdot e_{1} \wedge e_{3} \wedge e_{6} \wedge e_{7}
$$

The Ricci tensor $\left.\left.2 \cdot \operatorname{Ric}^{\nabla}(X, Y)=(X\lrcorner d T, Y\right\lrcorner * \omega^{3}\right)$ is a diagonal matrix

$$
\operatorname{Ric}^{\nabla}=\operatorname{diag}(-2,0,-2,0,0,-2,-2)
$$

and the scalar curvature becomes negative, $\mathrm{Scal}^{\nabla}=-8$. The symmetric tensor $T_{i m n} T_{j m n}$ is of diagonal form too,

$$
T_{i m n} T_{j m n}=\operatorname{diag}(4,0,4,4,4,4,4),
$$

and thus we obtain the Riemannian Ricci tensor

$$
\operatorname{Ric}_{i j}^{g}=\frac{1}{4} T_{i m n} T_{j m n}+\operatorname{Ric}_{i j}^{\nabla}=\operatorname{diag}(-1,0,-1,1,1,-1,-1) .
$$

Now we study the $\nabla$-parallel spinors. First of all we need the 4 -forms

$$
\begin{aligned}
& \frac{1}{4} d T+\frac{1}{2} \sigma^{T}=-e_{1} \wedge e_{3} \wedge e_{6} \wedge e_{7}+\left(e_{3} \wedge e_{4} \wedge e_{5} \wedge e_{6}-e_{1} \wedge e_{4} \wedge e_{5} \wedge e_{7}-e_{1} \wedge e_{3} \wedge e_{6} \wedge e_{7}\right), \\
& \frac{3}{4} d T-\frac{1}{2} \sigma^{T}=-3 e_{1} \wedge e_{3} \wedge e_{6} \wedge e_{7}-\left(e_{3} \wedge e_{4} \wedge e_{5} \wedge e_{6}-e_{1} \wedge e_{4} \wedge e_{5} \wedge e_{7}-e_{1} \wedge e_{3} \wedge e_{6} \wedge e_{7}\right) .
\end{aligned}
$$

LEMMA 6.1.

1) The 4-form $d T / 4+\sigma^{T} / 2$ acts in the spinor bundle as a symmetric endomorphism with eigenvalues $(2,-4,2,0,2,0,2,-4)$.

2) The 4 -form $3 \cdot d T / 4-\sigma^{T} / 2$ acts in the spinor bundle as a symmetric endomorphism with eigenvalues $(2,0,2,-4,2,-4,2,0)$. 
The proof of Lemma 6.1 is an easy computation in the spin representation of the 7-dimensional Clifford algebra.

COROLlaRY 6.2. There are four $\nabla$-parallel spinor fields on $M^{7}$. The torsion form acts trivially on any of these spinors, $T \cdot \Psi=0$.

Let $G$ be a discrete group of isometries acting on $M^{7}$ and preserving the $G_{2}$-structure $\omega^{3}$. Then $M^{7} / G$ admits a $G_{2}$-structure of type $\Lambda_{27}^{3}$.

COROLlary 6.3. If $M^{7} / G$ is a compact manifold and $\Psi$ is $\nabla$-harmonic, then

$$
6 \cdot \int_{M^{7} / G}\|\Psi\|^{2} \geq \int_{M^{7} / G}\|\nabla \Psi\|^{2} .
$$

Proof. Combining the Schrödinger-Lichnerowicz formula and Theorem 3.3 we obtain, in case of a $\nabla$-harmonic spinor, the equation

$$
\int_{M^{7} / G}\left(\|\nabla \Psi\|^{2}+\frac{1}{4}(d T \cdot \Psi, \Psi)+\frac{1}{2}\left(\sigma^{T} \cdot \Psi, \Psi\right)+\frac{1}{4} \operatorname{Scal}^{\nabla} \cdot\|\Psi\|^{2}\right)=0 .
$$

Since $\mathrm{Scal}^{\nabla}=-8$, the proof follows directly by Lemma 6.1 .

We now discuss a second example. The product $M^{7}=N^{6} \times \mathbb{R}^{1}$ of $\mathbb{R}^{1}$ by a 3 dimensional complex, solvable Lie group $N^{6}$ admits a left invariant metric such that the following structure equations hold (see [9]):

$$
\begin{gathered}
d e_{1}=0, \quad d e_{2}=0, \quad d e_{7}=0 \\
d e_{3}=e_{1} \wedge e_{3}-e_{2} \wedge e_{4}, \quad d e_{4}=e_{2} \wedge e_{3}+e_{1} \wedge e_{4}, \\
d e_{5}=-e_{1} \wedge e_{5}+e_{2} \wedge e_{6}, \quad d e_{6}=-e_{2} \wedge e_{5}-e_{1} \wedge e_{6} .
\end{gathered}
$$

A computation of the exterior products yields the formulas :

$$
d * \omega^{3}=0, \quad d \omega^{3}=2 \cdot e_{1} \wedge e_{3} \wedge e_{4} \wedge e_{7}-2 \cdot e_{1} \wedge e_{5} \wedge e_{6} \wedge e_{7} .
$$

In particular, the corresponding $G_{2}$-structure is cocalibrated and of pure type $\Lambda_{27}^{3}$,

$$
* d \omega^{3} \wedge \omega^{3}=0, \quad * d \omega^{3} \wedge * \omega^{3}=0 .
$$

The torsion tensor $T$ of the connection associated with the $G_{2}$-structure is given by $T=2 \cdot e_{2} \wedge e_{5} \wedge e_{6}-2 \cdot e_{2} \wedge e_{3} \wedge e_{4}, \quad d T=-4 \cdot e_{1} \wedge e_{2} \wedge e_{5} \wedge e_{6}-4 \cdot e_{1} \wedge e_{2} \wedge e_{3} \wedge e_{4}$.

We compute the scalar curvature $\operatorname{Scal}^{\nabla}=-16$ of the connection $\nabla$ and the 4 -forms

$$
\begin{aligned}
& \frac{3}{4} d T-\frac{1}{2} \sigma^{T}=-3 \cdot e_{1} \wedge e_{2} \wedge e_{5} \wedge e_{6}-3 \cdot e_{1} \wedge e_{2} \wedge e_{3} \wedge e_{4}+2 \cdot e_{3} \wedge e_{4} \wedge e_{5} \wedge e_{6} \\
& \frac{1}{4} d T+\frac{1}{2} \sigma^{T}=-e_{1} \wedge e_{2} \wedge e_{5} \wedge e_{6}-e_{1} \wedge e_{2} \wedge e_{3} \wedge e_{4}-2 \cdot e_{3} \wedge e_{4} \wedge e_{5} \wedge e_{6} .
\end{aligned}
$$


LEMMA 6.4.

1) In the spinor bundle the 4-forms $d T / 4+\sigma^{T} / 2$ acts in the spinor bundle as a symmetric endomorphism with eigenvalues $(4,4,-2,-2,-2,-2,0,0)$.

2) The 4-form $3 \cdot d T / 4-\sigma^{T} / 2$ acts as a symmetric endomorphism with eigenvalues $(4,4,2,2,2,2,-8,-8)$ in the spinor bundle.

COROLlary 6.5. There are two $\nabla$-parallel spinor fields on $M^{7}$. The torsion form acts trivially on any of these spinors, $T \cdot \Psi=0$.

Let $\mathrm{G}$ be a discrete group of isometries acting on $M^{\top}$ and preserving the $G_{2}$-structure $\omega^{3}$. Then $M^{7} / G$ admits a $G_{2}$-structure of type $\Lambda_{27}^{3}$.

Corollary 6.6. If $M^{7} / G$ is a compact manifold and $\Psi$ is $\nabla$-harmonic, then

$$
6 \cdot \int_{M^{7} / G}\|\Psi\|^{2} \geq \int_{M^{7} / G}\|\nabla \Psi\|^{2}
$$

We would like to mention that any hypersurface $M^{7} \subset \mathbb{R}^{8}$ admits a cocalibrated $G_{2^{-}}$ structure (see [11]). This structure is of pure type $\Lambda_{1}^{0}$ if and only if the hypersurface is umbilic. The pure type $\Lambda_{27}^{3}$ occurs if and only if the hypersurface is minimal. The function $\lambda$ is proportional to the mean curvature of the hypersurface (see [11]). Moreover, it turns out that in the decomposition

$$
T=-\frac{1}{6} \cdot \lambda \cdot \omega^{3}-\Gamma_{27}^{3}
$$

of the torsion tensor $T$ the 3 -form $\Gamma_{27}^{3}$ corresponds to the traceless part of the second fundamental form of the hypersurface via the $G_{2}$-isomorphism $S_{0}^{2}\left(\mathbb{R}^{7}\right)=\Lambda_{27}^{3}$. The torsion form is coclosed for hypersurfaces of constant mean curvature. In this case we obtain solutions of the equations in Theorem 5.1 such that $\delta^{g}(T)=0$.

7. Sasakian manifolds in dimension five. The case of the group $G_{2}$ and dimension $n=7$ discussed in detail fits into a more general approach described in the introduction. We study two further natural geometric structures: almost metric contact structures and almost hermitian structures. To begin with, let us consider the case of 5-dimensional Sasakian manifolds.

Proposition 7.1. Every Sasakian manifold $\left(M^{2 k+1}, g, \xi, \eta, \varphi\right)$ admits a unique metric connection with totally skew-symmetric torsion preserving the Sasakian structure :

$$
\nabla \xi=\nabla \eta=\nabla \varphi=0
$$

The connection $\nabla$ is given by

$$
g\left(\nabla_{X} Y, Z\right)=g\left(\nabla_{X}^{g} Y, Z\right)+\frac{1}{2}(\eta \wedge d \eta)(X, Y, Z), \quad T=\eta \wedge d \eta .
$$

The torsion form $T$ is $\nabla$-parallel and henceforth coclosed, $\delta^{g}(T)=0$. The 4-form $2 \cdot \sigma^{T}$ coincides with $d T$,

$$
2 \cdot \sigma^{T}=d T=d \eta \wedge d \eta
$$


Proof. The existence of the connection has been noticed in earlier papers (see e.g.[38]). The uniqueness will be proved in a more general context in Theorem 8.2.

We now consider a 5 -dimensional Sasakian manifold $M^{5}$ and orient it by the condition that the differential of the contact form is given by

$$
d \eta=2 \cdot\left(e_{1} \wedge e_{2}+e_{3} \wedge e_{4}\right) .
$$

Furthermore, we fix a spin structure. The endomorphism $\eta \wedge d \eta=2 \cdot\left(e_{1} \wedge e_{2}+e_{3} \wedge\right.$ $\left.e_{4}\right) \wedge e_{5}$ acts in the 5 -dimensional spin representation with eigenvalues $(-4,0,0,4)$. Consequently, the spinor bundle splits into two 1-dimensional and one 2-dimensional $\nabla$-parallel subbundles. The Clifford multiplication by $\xi$ preserves this decomposition of the spinor bundle and acts on the 1-dimensional bundles by multiplication by $i$, on the 2-dimensional bundle by multiplication by $-i$. Suppose that there exists a $\nabla$ parallel spinor $\Psi$. Then one of the subbundles under consideration admits a $\nabla$-parallel spinor

$$
\left.\nabla_{X}^{g} \Psi+\frac{1}{4}(X\lrcorner \eta \wedge d \eta\right) \cdot \Psi=0
$$

and the Riemannian Dirac operator for this spinor is given by the formula

$$
D^{g} \Psi+\frac{3}{4}(\eta \wedge d \eta) \cdot \Psi=0 .
$$

Let us first discuss the case that $\Psi$ belongs to one of the 1-dimensional subbundles defined by the algebraic equation $\xi \cdot \Psi=i \cdot \Psi$. In this case we apply Corollary 3.2

$$
\left.\frac{1}{2} \cdot(X\lrcorner d T\right) \cdot \Psi-\operatorname{Ric}^{\nabla}(X) \cdot \Psi=0
$$

as well as the following algebraic lemma.

LEMmA 7.2. The spinor $\Psi=(1,0,0,0)$ or $(0,0,0,1)$ belongs to the kernel of the endomorphism

$$
\sum_{1 \leq i<j<k \leq 5} t_{i j k} \cdot e_{i} \cdot e_{j} \cdot e_{j}+\sum_{i=1}^{5} x_{i} \cdot e_{i}
$$

in the 5-dimensional spin representation if and only if the following equations hold:

$$
\begin{gathered}
x_{1}=-t_{234}, \quad x_{2}=t_{134}, \quad x_{3}=-t_{124}, \quad x_{4}=t_{123}, \quad x_{5}=0, \\
t_{125}=-t_{345}, \quad t_{235}=-t_{145}, \quad t_{245}=t_{135} .
\end{gathered}
$$

Using these formulas we conclude $\operatorname{Ric}^{\nabla}=\operatorname{diag}(a, a, a, a, 0)$, where $2 \cdot a:=$ $d T\left(e_{1}, e_{2}, e_{3}, e_{4}\right)=d \eta \wedge d \eta\left(e_{1}, e_{2}, e_{3}, e_{4}\right)=8$. In particular, we obtain

$$
\operatorname{Ric}^{g}-\operatorname{diag}(2,2,2,2,4)=\operatorname{Ric}^{g}-\frac{1}{4} T_{i m n} T_{j m n}=\operatorname{Ric}^{\nabla},
$$

i.e., $\operatorname{Ric}^{g}=\operatorname{diag}(6,6,6,6,4)$. A proof similar to the proof of the existence of Killing spinors on Einstein-Sasakian manifolds (see [18]) shows that this condition is the only 
integrability condition for $\nabla$-parallel spinors in the 1-dimensional subbundles. The endomorphisms act in the spinor bundle

$$
\frac{3}{4} d T-\frac{1}{2} \sigma^{T}+\frac{1}{4} \mathrm{Scal}^{\nabla}=4 \cdot\left(e_{1} \cdot e_{2} \cdot e_{3} \cdot e_{4}+1\right)=\frac{1}{4} d T+\frac{1}{2} \sigma^{T}+\frac{1}{4} \mathrm{Scal}^{\nabla}
$$

with eigenvalues 0 and 4 . Corollary 3.2 and Theorem 3.3 yield

Theorem 7.3. Let $\left(M^{5}, g, \xi, \eta, \varphi\right)$ be a simply connected 5-dimensional Sasakian spin manifold and consider the unique linear connection $\nabla$ with totally skewsymmetric torsion preserving the Sasakian structure. There exists a $\nabla$-parallel spinor in the subbundle defined by the algebraic equation $\xi \cdot \Psi=i \cdot \Psi$ if and only if the Riemannian Ricci tensor of $M^{5}$ has the eigenvalues $(6,6,6,6,4) . A \nabla$-parallel spinor of this algebraic type is an eigenspinor of the Riemannian Dirac operator, $D^{g} \Psi= \pm 3 \cdot \Psi$. In case $M^{5}$ is compact, any $\nabla$-harmonic spinor $\Psi$ is $\nabla$-parallel.

EXAMPLE 7.4. Sasakian manifolds with the described form of the Ricci tensor can be constructed - for example - as bundles over 4-dimensional Kähler-Einstein manifolds with positive scalar curvature. Indeed, consider a simply connected Kähler-Einstein manifold $\left(N^{4}, J, g^{*}\right)$ with scalar curvature Scal ${ }^{*}=32$. Then there exists an $S^{1}$-bundle $M^{5} \rightarrow N^{4}$ as well as a Sasakian structure on $M^{5}$ such that the Ricci tensor has the eigenvalues $\operatorname{Ric}^{g}=\operatorname{diag}(6,6,6,6,4)$ (see [20]). More general, the Tanno deformation of an arbitrary 5-dimensional Einstein-Sasakian structure yields for a special value of deformation parameter examples of Sasakian manifolds satisfying the condition of Theorem 7.3 (see Example 9.3). The Einstein-Sasakian manifolds constructed recently in [6] admit $\nabla$-parallel spinors with respect to a Tanno deformation of the Sasakian structure.

We discuss the case that the $\nabla$-parallel spinor field $\Psi$ belongs to the 2-dimensional subbundle defined by the algebraic equation $d \eta \cdot \Psi=0$. A spinor field of this type is a Riemannian harmonic spinor. Let us again compute the Ricci tensor Ric ${ }^{\nabla}$ :

LEMmA 7.5. The spinor $\Psi=(0,1,0,0)$ belongs to the kernel of the endomorphism

$$
\sum_{1 \leq i<j<k \leq 5} t_{i j k} \cdot e_{i} \cdot e_{j} \cdot e_{j}+\sum_{i=1}^{5} x_{i} \cdot e_{i}
$$

in the 5-dimensional spin representation if and only if the following equations hold:

$$
\begin{gathered}
x_{1}=t_{234}, \quad x_{2}=-t_{134}, \quad x_{3}=t_{124}, \quad x_{4}=-t_{123}, \quad x_{5}=0, \\
t_{125}=t_{345}, \quad t_{235}=t_{145}, \quad t_{245}=-t_{135} .
\end{gathered}
$$

In this case we obtain

$$
\operatorname{Ric}^{\nabla}=\operatorname{diag}(-4,-4,-4,-4,0), \quad \operatorname{Ric}^{g}=\operatorname{diag}(-2,-2,-2,-2,4) .
$$

We compute the endomorphisms acting in the spinor bundle :

$$
\frac{3}{4} d T-\frac{1}{2} \sigma^{T}+\frac{1}{4} \mathrm{Scal}^{\nabla}=4 \cdot\left(e_{1} \cdot e_{2} \cdot e_{3} \cdot e_{4}-1\right)=\frac{1}{4} d T+\frac{1}{2} \sigma^{T}+\frac{1}{4} \mathrm{Scal}^{\nabla} .
$$


The Clifford product $e_{1} \cdot e_{2} \cdot e_{3} \cdot e_{4}$ acts in the 2-dimensional subbundle of the spin bundle as the identity. Corollary 3.2 and Theorem 3.3 yield

TheOREM 7.6. Let $\left(M^{5}, g, \xi, \eta, \varphi\right)$ be a 5-dimensional Sasakian spin manifold and consider the unique linear connection $\nabla$ with totally skew-symmetric torsion preserving the Sasakian structure. If there exists a $\nabla$-parallel spinor in the subbundle defined by the algebraic equation $d \eta \cdot \Psi=0$, then the Riemannian Ricci tensor of $M^{5}$ has the eigenvalues $(-2,-2,-2,-2,4)$. Any $\nabla$-parallel spinor in this 2-dimensional subbundle satisfies the equations

$$
\nabla_{\xi}^{g} \Psi=0, \quad \nabla_{X}^{g} \Psi=\frac{1}{2} \varphi(X) \cdot \xi \cdot \Psi=-\frac{i}{2} \varphi(X) \cdot \Psi, \quad d \eta \cdot \Psi=0 .
$$

In particular, it is harmonic with respect to the Riemannian connection. Any $\nabla$-harmonic spinor $\Psi$ on a compact manifold $M^{5}$ satisfying the algebraic condition $d \eta \cdot \Psi=0$ is $\nabla$-parallel.

EXAMPLE 7.7. In $\mathbb{R}^{5}$ we consider the 1-forms

$$
\begin{gathered}
e_{1}=\frac{1}{2} \cdot d x_{1}, \quad e_{2}=\frac{1}{2} \cdot d y_{1}, \quad e_{3}=\frac{1}{2} \cdot d x_{2}, \quad e_{4}=\frac{1}{2} \cdot d y_{2}, \\
e_{5}=\eta=\frac{1}{2} \cdot\left(d z-y_{1} \cdot d x_{1}-y_{2} \cdot d x_{2}\right) .
\end{gathered}
$$

We obtain a Sasakian manifold (see [4]) and it is not hard to see that it admits $\nabla$-parallel spinors of type $d \eta \cdot \Psi=0$. The Sasakian structure arises from left invariant vector fields on a 5-dimensional Heisenberg group.

Sasakian manifolds with $\nabla$-parallel spinors of type $F \cdot \Psi=0$ may be constructed as bundles over the 4-dimensional torus. Indeed, suppose that the Sasakian structure is regular. Then $M^{5}$ is a $S^{1}$-bundle over $N^{4}$. The spinor field $\Psi$ is projectable and induces a parallel spinor field $\Psi^{*}$ in the negative spinor bundle $\Sigma^{-}\left(N^{4}\right)$ over $N^{4}$ (see [41]). Consequently, $N^{4}$ is a selfdual, Ricci-flat Kähler manifold. On the other hand, the endomorphism $\varphi$ projects too and we obtain a second integrable, but in general not parallel positive complex structure. There is only one possibility for $N^{4}$, the torus $T^{4}$. In a forthcoming paper (see [17]) we will study these $\nabla$-parallel spinor even for normal almost contact metric structures in more details. In particular it turns out that the Example 7.7 is (locally) the only Sasakian space with $\nabla$-parallel spinors of type $d \eta \cdot \Psi=0$.

8. Almost contact connections with totally skew-symmetric torsion. Let us discuss the latter results from a more general point of view and consider an almost contact metric manifold $\left(M^{2 k+1}, g, \xi, \eta, \varphi\right)$, i.e., a Riemannian manifold equipped with a 1-form $\eta$, a $(1,1)$-tensor $\varphi$ and a vector field $\xi$ dual to $\eta$ with respect to the metric $g$ such that the following compatibility conditions are satisfied (see [4]):

$\eta(\xi)=1, \quad \varphi^{2}=-\mathrm{Id}+\eta \otimes \xi, \quad g(\varphi(X), \varphi(Y))=g(X, Y)-\eta(X) \cdot \eta(Y), \quad \varphi(\xi)=0$.

Let us introduce the fundamental form $F(X, Y):=g(X, \varphi(Y))$ as well as the Nijenhuis tensor

$$
N(X, Y):=[\varphi(X), \varphi(Y)]+\varphi^{2}[X, Y]-\varphi[\varphi(X), Y]-\varphi[X, \varphi(Y)]+d \eta(X, Y) \cdot \xi
$$




$$
N^{2}(X, Y):=d \eta(\varphi(X), Y)+d \eta(X, \varphi(Y)) .
$$

We recall some notions describing the different types of almost contact metric structures. If $2 \cdot F=d \eta$, then we have a contact metric structure, if $N=0$, we have a normal contact structure. A $K$-contact structure is a contact metric structure such that the vector field $\xi$ is a Killing vector field. If the structure is normal and $\mathrm{K}$ contact, then it is a Sasaki structure (a complete classification of almost contact metric structures is presented in $[2,7,8])$. The Nijenhuis tensor of type $(0,3)$ is given by $N(X, Y, Z)=g(N(X, Y), Z)$. We have the following general identities $[4,7,8]$ :

$$
\begin{gathered}
2 \cdot g\left(\left(\nabla_{X}^{g} \varphi\right)(Y), Z\right)=d F(X, \varphi(Y), \varphi(Z))-d F(X, Y, Z)+N(Y, Z, \varphi(X)) \\
+\eta(X) \cdot N^{2}(Y, Z)+\eta(Z) \cdot d \eta(\varphi(Y), X)+\eta(Y) \cdot d \eta(X, \varphi(Z)), \\
g\left(\left(\nabla_{X}^{g} \varphi\right)(Y), Z\right)+g\left(\left(\nabla_{X}^{g} \varphi\right)(\varphi(Y)), \varphi(Z)\right)=\eta(Y) \cdot\left(\nabla_{X}^{g} \eta\right)(\varphi(Z)) \\
-\eta(Z) \cdot\left(\nabla_{X}^{g} \eta\right)(\varphi(Y)), \\
g\left(\left(\nabla_{X}^{g} \varphi\right)(\varphi(Y)), \xi\right)=\left(\nabla_{X}^{g} \eta\right)(Y)=g\left(\nabla_{X}^{g} \xi, Y\right), \\
N(X, Y, Z)=-N(\varphi(X), \varphi(Y), Z)+\eta(X) \cdot N(\xi, Y, Z)+\eta(Y) \cdot N(X, \xi, Z) \\
=-N(\varphi(X), Y, \varphi(Z))+\eta(Z) \cdot N(\xi, X, Y)-\eta(X) \cdot N(\xi, \varphi(Y), \varphi(Z)) .
\end{gathered}
$$

Finally, we introduce the forms

$$
\begin{aligned}
d F^{-}(X, Y, Z):= & d F(X, \varphi(Y), \varphi(Z))+d F(\varphi(X), Y, \varphi(Z)) \\
& +d F(\varphi(X), \varphi(Y), Z)-d F(X, Y, Z), \\
d^{\varphi} F(X, Y, Z):= & -d F(\varphi(X), \varphi(Y), \varphi(Z)),
\end{aligned}
$$

and a direct consequence of the definitions is the following

Proposition 8.1. On any almost contact metric manifold the identities hold:

1) $d F^{-}(X, Y, Z)=-N(X, Y, \varphi(Z))-N(Y, Z, \varphi(X))-N(Z, X, \varphi(Y))$,

2) $N(X, Y)=\left(\nabla_{\varphi(X)}^{g} \varphi\right)(Y)-\left(\nabla_{\varphi(Y)}^{g} \varphi\right)(X)+\left(\nabla_{X}^{g} \varphi\right)(\varphi(Y))-\left(\nabla_{Y}^{g} \varphi\right)(\varphi(X))$

$$
-\eta(Y) \cdot \nabla_{X}^{g} \xi+\eta(X) \cdot \nabla_{Y}^{g} \xi
$$

A linear connection $\nabla$ is said to be an almost contact connection if it preserves the almost contact structure :

$$
\nabla g=\nabla \eta=\nabla \varphi=0 .
$$

THEOREM 8.2. Let $\left(M^{2 k+1}, g, \xi, \eta, \varphi\right)$ be an almost contact metric manifold. The following conditions are equivalent:

1) The Nijenhuis tensor $N$ is skew-symmetric and $\xi$ is a Killing vector field.

2) There exists an almost contact linear connection $\nabla$ with totally skewsymmetric torsion tensor $T$.

Moreover, this connection is unique and determined by

$$
g\left(\nabla_{X} Y, Z\right)=g\left(\nabla_{X}^{g} Y, Z\right)+\frac{1}{2} T(X, Y, Z),
$$

where $\nabla^{g}$ is the Levi-Civita connection and the torsion $T$ is defined by

$$
\left.T=\eta \wedge d \eta+d^{\varphi} F+N-\eta \wedge(\xi\lrcorner N\right) .
$$


Proof. Let us assume that such a connection exists. Then

$$
0=g\left(\nabla_{X}^{g} \xi, Z\right)+\frac{1}{2} T(X, \xi, Z)
$$

holds and the skew-symmetry of $T$ yields that $\xi$ is a Killing vector field, $d \eta=\xi\lrcorner$ $T, \xi\lrcorner d \eta=0$ and

$$
\begin{aligned}
& T(\varphi(X), \varphi(Y), Z)-T(X, Y, Z)+T(\varphi(X), Y, \varphi(Z))+T(X, \varphi(Y), \varphi(Z)) \\
= & -N(X, Y, Z) .
\end{aligned}
$$

The latter formula shows that $N$ is skew-symmetric. Since $\varphi$ is $\nabla$-parallel, we can express the Riemannian covariant derivative of $\varphi$ by the torsion form:

$$
T(X, Y, \varphi(Z))+T(X, \varphi(Y), Z)=-2 \cdot g\left(\left(\nabla_{X}^{g} \varphi\right) Y, Z\right) .
$$

Taking the cyclic sum in the above equality, we obtain

$$
\sigma_{X, Y, Z} T(X, Y, \varphi(Z))=-\sigma_{X, Y, Z} g\left(\left(\nabla_{X}^{g} \varphi\right) Y, Z\right) .
$$

We use Proposition 8.1 as well as the identity preceding it to get

$$
-\sigma_{X, Y, Z} T(X, Y, \varphi(Z))=\sigma_{X, Y, Z} g\left(\left(\nabla_{X}^{g} \varphi\right) Y, Z\right)=-d F(X, Y, Z) .
$$

Adding this result to the formula expressing the Nijenhuis tensor $N$ by the torsion $T$, some calculations yield

$$
T(\varphi(X), \varphi(Y), \varphi(Z))=d F(X, Y, Z)-N(X, Y, \varphi(Z))-\eta(Z) \cdot N^{2}(X, Y) .
$$

By replacing $X, Y, Z$ by $\varphi(X), \varphi(Y), \varphi(Z)$ and using the symmetry property of the Nijenhuis tensor mentioned before Proposition 8.1, we obtain the formula for the torsion tensor $T$. For the converse, suppose that the almost contact structure has the properties 1) and define the connection $\nabla$ by the formulas in 2). Clearly $T$ is skew-symmetric and $\xi\lrcorner T=d \eta=2 \nabla^{g} \eta$. Since $\xi$ is a Killing vector field, we conclude $\nabla g=\nabla \xi=0$. Furthermore, using the conditions 1) and Proposition 8.1, we obtain $\xi\lrcorner d F=N^{2}$. Finally we have to prove that $\nabla \varphi=0$. This follows by straightforward computations using the relation between $\nabla \varphi$ and the torsion tensor $T$, Proposition 8.1 as well as the following lemma.

LEMMA 8.3. Let $\left(M^{2 k+1}, g, \xi, \eta, \varphi\right)$ be an almost contact metric manifold with a totally skew-symmetric Nijenhuis tensor $N$ and Killing vector field $\xi$. Then the following equalities hold:

$$
\begin{gathered}
\left.\nabla_{\xi}^{g} \xi=\xi\right\lrcorner d \eta=0, \\
\begin{aligned}
N(\varphi(X), Y, \xi)= & N(X, \varphi(Y), \xi) \\
= & N^{2}(X, Y)=d F(X, Y, \xi)=-d F(\varphi(X), \varphi(Y), \xi) .
\end{aligned}
\end{gathered}
$$

Proof. The identities in and before Proposition 8.1 imply

$0=N(X, \xi, \xi)=\left(\nabla_{\xi}^{g} \varphi\right)(\xi), \quad 0=N(\xi, X, X)=\left(\nabla_{X}^{g} \eta\right)(X)-\left(\nabla_{\varphi(X)}^{g} \eta\right)(\varphi(X))$ 


$$
N(X, Y, \xi)=d \eta(X, Y)-d \eta(\varphi(X), \varphi(Y)) .
$$

Hence, the first two equalities follow. We take the cyclic sum in the second idendity before Proposition 8.1 and put $X=\xi$ to obtain

$$
-d F(\xi, Y, Z)+g\left(\left(\nabla_{\xi}^{g} \varphi\right)(\varphi(Y)), \varphi(Z)\right)=-\left(\nabla_{Y}^{g} \eta\right)(\varphi(Z))+\left(\nabla_{Z}^{g} \eta\right)(\varphi(Y)) .
$$

On the other hand, using again the general formulas we calculate that

$$
-d F(\xi, \varphi(Y), \varphi(Z))=g\left(\left(\nabla_{\xi}^{g} \varphi\right)(\varphi(Y)) \cdot \varphi(Z)\right)+\left(\nabla_{\varphi(Y)}^{g} \eta\right)(Z)-\left(\nabla_{\varphi(Z)}^{g} \eta\right)(Y) .
$$

Summing up the latter two equalities we obtain the last equalities in the lemma since $\xi$ is a Killing vector field.

We discuss these results for some special contact structures.

THEOREM 8.4. Let $\left(M^{2 k+1}, g, \xi, \eta, \varphi\right)$ be an almost contact metric manifold with totally skew-symmetric Nijenhuis tensor $N$. Then the condition $d F=0$ implies $N=$ 0 .

1) A contact metric structure $(2 \cdot F=d \eta)$ admits an almost contact connection with totally skew-symmetric torsion if and only if it is Sasakian. In this case, the connection is unique, its torsion is given by

$$
T=\eta \wedge d \eta
$$

and $T$ is parallel, $\nabla T=0$.

2) A normal $(N=0)$ almost contact structure admits a unique almost contact connection with totally skew-symmetric torsion if and only if $\xi$ is a Killing vector field. The torsion $T$ is then given by

$$
T=\eta \wedge d \eta+d^{\varphi} F .
$$

Proof. If $d F=0$, Lemma 8.3 implies that $\left.N^{2}=\xi\right\lrcorner N=0$. Then Proposition 8.1 leads to $0=d F^{-}(X, Y, Z)=-3 \cdot N(\varphi(X), Y, Z)$. The assertion that $\nabla T=0$ in a Sasakian manifold follows by direct verification.

9. Almost contact structures, parallel spinors and holonomy group. Let $\left(M^{2 k+1}, g, \xi, \eta, \varphi\right)$ be a $(2 k+1)$-dimensional almost contact metric manifold with totally skew-symmetric Nijenhuis tensor $N$ and Killing vector $\xi$ and denote by $\nabla$ the unique almost contact connection with a totally skew-symmetric torsion (Theorem 8.2). Since $\nabla \xi=0$ the (restricted) holonomy group $\mathrm{Hol}^{\nabla}$ of $\nabla$ is contained in $U(k)$. This group cannot occur as the isotropy group of any spinor. The spinor bundle $\Sigma$ of a contact spin manifold decomposes under the action of the fundamental form $F$ into the sum (see [20])

$$
\Sigma=\Sigma_{0} \oplus \ldots \Sigma_{k}, \quad \operatorname{dim}\left(\Sigma_{r}\right)=\left(\begin{array}{l}
k \\
r
\end{array}\right) .
$$

The isotropy group of a spinor of type $\Sigma_{0}$ or $\Sigma_{k}$ coincides with the subgroup $S U(k) \subset$ $U(k)$. Consequently, there exists locally a $\nabla$-parallel spinor of type $\Sigma_{0}, \Sigma_{k}$ if and 
only if $\mathrm{Hol}^{\nabla}$ is contained in $S U(k)$. We shall express this condition in terms of the curvature of $\nabla$. The group $\mathrm{Hol}^{\nabla}$ is contained in $S U(k)$ if and only if the 2-form

$$
\varrho^{\nabla}(X, Y):=\frac{1}{2} \sum_{i=1}^{2 k+1} R^{\nabla}\left(X, Y, e_{i}, \varphi\left(e_{i}\right)\right)
$$

vanishes, $\varrho^{\nabla}=0$. Let us introduce the torsion 1-form $\omega^{\nabla}$ as well as the 2-form $\lambda^{\nabla}$ by

$$
\omega^{\nabla}(X):=-\frac{1}{2} \sum_{i=1}^{2 k+1} T\left(X, e_{i}, \varphi\left(e_{i}\right)\right), \quad \lambda^{\nabla}(X, Y):=\frac{1}{2} \sum_{i=1}^{2 k+1} d T\left(X, Y, e_{i}, \varphi\left(e_{i}\right)\right) .
$$

Proposition 9.1. Let $\left(M^{2 k+1}, g, \xi, \eta, \varphi\right)$ be a $(2 k+1)$-dimensional almost contact metric manifold with totally skew-symmetric Nijenhuis tensor $N$ and Killing vector $\xi$. Let $\nabla$ be the unique almost contact connection with totally skew-symmetric torsion. Then one has

$$
\varrho^{\nabla}(X, Y)=\operatorname{Ric}^{\nabla}(X, \varphi(Y))-\left(\nabla_{X} \omega^{\nabla}\right)(Y)+\frac{1}{4} \lambda^{\nabla}(X, Y) .
$$

Proof. We follow the scheme in [36], Section 3, and use the curvature properties of $\nabla$ from Section 2 to calculate $\lambda^{\nabla}(X, Y)$ :

$$
-2\left(\nabla_{X} \omega\right)(Y)+2\left(\nabla_{Y} \omega\right)(X)+2 \sum_{i=1}^{2 k+1}\left(\sigma^{T}\left(X, Y, e_{i}, \varphi\left(e_{i}\right)\right)-\left(\nabla_{\varphi\left(e_{i}\right)} T\right)\left(X, Y, e_{i}\right)\right) .
$$

The first Bianchi identity for $\nabla$ together with the latter identity implies

$$
\begin{aligned}
& 4 \rho^{\nabla}(X, Y)+2 \operatorname{Ric}^{\nabla}(Y, \varphi(X))-2 \operatorname{Ric}^{\nabla}(X, \varphi(Y)) \\
= & \lambda^{\nabla}(X, Y)-2\left(\nabla_{X} \omega\right)(Y)+2\left(\nabla_{Y} \omega\right)(X) .
\end{aligned}
$$

Using the relation between the curvature tensors of $\nabla$ and $\nabla^{g}$, we obtain

$$
\operatorname{Ric}^{\nabla}(Y, \varphi(X))+\operatorname{Ric}^{\nabla}(X, \varphi(Y))=\left(\nabla_{X} \omega^{\nabla}\right)(Y)+\left(\nabla_{Y} \omega^{\nabla}\right)(X) .
$$

The last two equalities lead to the desired formula.

We apply Proposition 9.1 in case of a Sasakian manifold.

Theorem 9.2. Let $\left(M^{2 k+1}, g, \xi, \eta, \varphi, \nabla\right)$ be a simply connected $(2 k+1)$ dimensional Sasakian spin manifold and $\nabla$ be the unique almost contact connection with totally skew-symmetric torsion. Then there exists a $\nabla$-parallel spinor of type $\Sigma_{0}$ or $\Sigma_{k}$ if and only if the Ricci tensor is given by the formula :

$$
\operatorname{Ric}^{\nabla}=4 \cdot(k-1) \cdot(g-\eta \otimes \eta) .
$$

This condition is equivalent to

$$
\operatorname{Ric}^{g}=2 \cdot(2 k-1) \cdot g-2 \cdot(k-1) \cdot \eta \otimes \eta .
$$

Proof. On a Sasakian manifold $T=\eta \wedge d \eta=2 \cdot \eta \wedge F$ and $\nabla T=0$, where $F(X, Y)=$ $g(X, \varphi(Y))$ is the fundamental form of the Sasakian structure. Consequently, we calculate that

$$
\nabla\left(\omega^{\nabla}\right)=0, \quad \lambda^{\nabla}=16 \cdot(1-k) F, \quad \sum_{i=1}^{2 k+1} g\left(T\left(X, e_{i}\right), T\left(Y, e_{i}\right)\right)=8 \cdot g+8 \cdot(k-1) \eta \otimes \eta,
$$


and the proof follows from Proposition 9.1.

REMARK 9.3. Sasakian manifolds with the prescribed form of the Ricci tensor admit Sasakian quasi-Killing spinors of type $( \pm 1 / 2, b)$ (see [20], Theorem 6.3). The Tanno deformation of an Einstein-Sasakian manifold defined by the formulas

$$
\tilde{\varphi}:=\varphi, \quad \tilde{\xi}:=a^{2} \cdot \xi, \quad \tilde{\eta}:=a^{-2} \cdot \eta: \quad \tilde{g}:=a^{-2} \cdot g+\left(a^{-4}-a^{-2}\right) \cdot \eta \otimes \eta
$$

yields for the parameter $a^{2}:=2 k /(k+1)$ a Sasakian manifold satisfying the condition of Theorem 9.2 and vice versa (see [20], Lemma 6.7 and Lemma 6.8).

10. Almost hermitian connections with totally skew-symmetric torsion. In this section we study connections with totally skew-symmetric torsion and preserving an almost complex structure. These exist, for example, for nearly Kähler manifolds. In dimension $n=6$ nearly Kähler manifolds have special properties and they are precisely the 6-dimensional manifolds admitting real Killing spinors (see [30] and $[31])$.

THEOREM 10.1. Let $\left(M^{2 n}, g, J\right)$ be a $2 n$-dimensional almost complex manifold. Then there exists a linear connection with totally skew-symmetric torsion preserving the hermitian structure $(g, J)$ if and only if the Nijenhuis tensor $N(X, Y, Z):=$ $g(N(X, Y), Z)$ is a 3-form. In this case the connection is unique and is determined by

$$
T(X, Y, Z)=-d \Omega(J(X), J(Y), J(Z))+N(X, Y, Z),
$$

where $\Omega$ is the Kähler form.

Proof. The result can be derived from the considerations in [25] or from Proposition 4.1. We sketch a direct proof. Since $\nabla g=\nabla J=0$, we have

$$
\begin{aligned}
& T(J(X), J(Y), Z)-T(X, Y, Z)+T(J(X), Y, J(Z))+T(X, J(Y), J(Z)) \\
= & -N(X, Y, Z)
\end{aligned}
$$

which shows that $N$ is a 3 -form. The formula for the torsion form follows from the following identities on an almost complex manifold with skew symmetric tensor $N$.

$$
\begin{aligned}
2 \cdot g\left(\left(\nabla_{X}^{g} J\right) Y, Z\right)= & d \Omega(X, J(Y), J(Z))-d \Omega(X, Y, Z)+N(Y, Z, J(X)) \\
d \Omega^{-}(X, Y, Z):= & d \Omega(X, J(Y), J(Z))-d \Omega(X, Y, Z)+d \Omega(J(X), J(Y), Z) \\
& +d \Omega(J(X), Y, J(Z))=-3 \cdot N(J(X), Y, Z) .
\end{aligned}
$$

Corollary 10.2. On an almost Kähler manifold there does not exist a hermitian connection with totally skew-symmetric torsion.

Corollary 10.3. On any nearly Kähler manifold the torsion form $T$ is $\nabla$-parallel and henceforth coclosed, $\delta^{g}(T)=0$.

Proof. If $\left(M^{2 n}, g, J\right)$ is a nearly Kähler manifold, then

$$
4 \cdot d \Omega(X, Y, Z)=-d \Omega^{-}(X, Y, Z)=3 \cdot N(J(X), Y, Z)
$$


and Theorem 10.1 yields that $4 \cdot T=N$. Moreover, $\nabla$ is the characteristic connection considered by Gray [28] and $T$ is $\nabla$-parallel, $\nabla T=\nabla N=0$ (see e.g. [37, 3]).

We compute the Ricci tensor $\operatorname{Ric}^{\nabla}$ for a 6-dimensional nearly Kähler spin manifold.

Proposition 10.4. On a 6-dimensional nearly Kähler manifold with nonvanishing Nijenhuis tensor $N \neq 0$ we have

$$
T_{i m n} T_{j m n}=2 \cdot a \cdot g_{i j}, \quad \operatorname{Ric}^{g}=\frac{5}{2} \cdot a \cdot g, \quad \operatorname{Ric}^{\nabla}=2 \cdot a \cdot g .
$$

The 4-form $2 \cdot \sigma^{T}$ coincides with $d T$,

$$
2 \cdot \sigma^{T}=d T=a \cdot(\Omega \wedge \Omega) .
$$

Proof. We recall (see [28]) that any 6-dimensional nearly Kähler manifold is Einstein and of constant type, i.e.

$$
\operatorname{Ric}^{g}=\frac{5}{2} \cdot a \cdot g, \quad\left\|\left(\nabla_{X}^{g} J\right) Y\right\|^{2}=\frac{1}{2} \cdot a \cdot\left(\|X\|^{2} \cdot\|Y\|^{2}-g^{2}(X, Y)-g^{2}(X, J(Y))\right)
$$

where $a:=\mathrm{Scal}^{g} / 15$ is a positive constant. Polarizing the latter equality und using the identity $4 \cdot J\left(\nabla_{X}^{g} J\right) Y=-N(X, Y)=-4 \cdot T(X, Y)$ we get

$$
T_{i m n} T_{j m n}=2 \cdot a \cdot g_{i j}, \quad 2 \cdot \sigma^{T}=a \cdot \Omega \wedge \Omega .
$$

We calculate $\operatorname{Ric}_{i j}^{\nabla}=\operatorname{Ric}_{i j}^{g}-\frac{1}{4} \cdot T_{i m n} T_{j m n}=2 \cdot a \cdot g_{i j}$ and the result follows.

We consider again the general almost complex case. Let $\left(M^{2 n}, g, J\right)$ be an almost complex manifold with totally skew-symmetric tensor $N$. Then $M^{2 n}$ is of type $G_{1}$ according to Gray-Hervella classification (see [29]). Denote by $\nabla$ the unique hermitian connection with totally skew-symmetric torsion $T$ described in Theorem 9.1. The Ricci form of $\nabla$ is defined by

$$
\varrho^{\nabla}(X, Y)=\frac{1}{2} \sum_{i=1}^{2 n} R^{\nabla}\left(X, Y, e_{i}, J\left(e_{i}\right)\right) .
$$

The holonomy group $\mathrm{Hol}^{\nabla}$ of $\nabla$ is contained in $S U(n)$ if and only if $\varrho^{\nabla}=0$. We define the Lee form $\theta$ and the tensor $\lambda^{\omega}$ by

$$
\theta(X)=-\frac{1}{2} \sum_{i=1}^{2 n} T\left(J(X), e_{i}, J\left(e_{i}\right)\right), \quad \lambda^{\omega}(X, Y)=\sum_{i=1}^{2 n} d T\left(X, Y, e_{i}, J\left(e_{i}\right)\right) .
$$

We remark that the formula (3.16) in [36] holds in the general case of a $G_{1}$-manifold,

$$
\varrho^{\nabla}(X, Y)=\operatorname{Ric}^{\nabla}(X, J(Y))+\left(\nabla_{X} \theta\right) J(Y)+\frac{1}{4} \lambda^{\omega}(X, Y) .
$$

THEOREM 10.5. Let $\left(M^{2 n}, g, J, \nabla\right)$ be an almost hermitian manifold of type $G_{1}$ with its unique linear connection $\nabla$ with totally skew-symmetric torsion. Then Hol $\nabla \subset$ $S U(n)$ if and only if

$$
0=\operatorname{Ric}^{\nabla}(X, J(Y))+\left(\nabla_{X} \theta\right) J(Y)+\frac{1}{4} \lambda^{\omega}(X, Y)
$$


Corollary 10.6. Let $\left(M^{2 n}, g, J, \nabla\right)$ be a nearly Kähler manifold. Then $\mathrm{Hol}^{\nabla}$ is contained in $S U(n)$ if and only if

$$
\operatorname{Ric}^{\nabla}(X, Y)=\frac{1}{4} \lambda^{\omega}(X, J(Y))
$$

If the manifold is not Kähler and the dimension is 6 , then the above condition is always satisfied.

Proof. In the nearly Kähler case the torsion form $T$ is $\nabla$-parallel and therefore $\theta$ is parallel too, $\nabla \theta=0$. In the 6-dimensional strictly nearly Kähler case the condition of the Corollary 10.6 is a consequence of Proposition 10.4 .

Finally we study in detail the $\nabla$-parallel and $\nabla$-harmonic spinor fields on a 6dimensional nearly Kähler manifold $M^{6}$. The spinor bundle $\Sigma\left(M^{6}\right)$ splits into the 1-dimensional subbundles $E^{ \pm} \subset \Sigma^{ \pm}\left(M^{6}\right)$ of the spinor bundle defined by the equation

$$
J(X) \cdot \Psi^{ \pm}=\mp i \cdot X \cdot \Psi^{ \pm}
$$

as well as their orthogonal complements. The connection $\nabla$ preserves this decomposition. We discuss the integrability condition (see Corollary 3.2) for the existence of a $\nabla$-parallel spinor. A purely algebraic computation in the 6-dimensional spin representation proves the following

LEMMA 10.7. A spinor $\Psi^{ \pm}$satisfies the equation

$$
\left.\frac{1}{2}(X\lrcorner d T\right) \cdot \Psi^{ \pm}-\operatorname{Ric}^{\nabla}(X) \cdot \Psi^{ \pm}=0
$$

for all vectors $X$ if and only if it belongs to $E^{ \pm}$. The endomorphism

$$
\begin{gathered}
\frac{3}{4} d T-\frac{1}{2} \sigma^{T}+\frac{1}{4} \mathrm{Scal}^{\nabla}=\frac{1}{4} d T+\frac{1}{2} \sigma^{T}+\frac{1}{4} \mathrm{Scal}^{\nabla} \\
=a \cdot\left(e_{1} \cdot e_{2} \cdot e_{3} \cdot e_{4}+e_{1} \cdot e_{2} \cdot e_{5} \cdot e_{6}+e_{3} \cdot e_{4} \cdot e_{5} \cdot e_{6}+3\right)
\end{gathered}
$$

acts in the spinor modules $\Delta_{6}^{ \pm}$with eigenvalues $(0,4 a, 4 a, 4 a)$.

Consequently, Corollary 3.2 and Theorem 3.3 yield the following results :

TheOREM 10.8. Let $\left(M^{6}, g, J\right)$ be a 6-dimensional nearly Kähler spin manifold and let $\nabla$ be the unique linear connection with totally skew-symmetric torsion preserving the nearly Kähler structure. Then there exist two $\nabla$-parallel spinors. These spinors are sections in the subbundles $E^{ \pm}$. If $M^{6}$ is compact, then every $\nabla$-harmonic spinor is $\nabla$-parallel.

REMARK 10.9. A 6-dimensional nearly Kähler manifold admits two Killing spinors with respect to the Levi-Civita connection (see [30]) and these spinor fields are the $\nabla$-parallel spinors. 


\section{REFERENCES}

[1] T. Ackermann, J. Tolksdorf, A generalized Lichnerowicz formula, the Wodzicki residue and gravity, Journ. Geom. Phys., 19 (1996), pp. 143-150.

[2] V. Aleksiev, G. Ganchev, On the classification of the almost contact metric manifolds, Mathematics and education in mathematics, Proc. 15th Spring Conf., Sunny Beach/Bulg. 1986, pp. 155-161.

[3] F. Belgun, A. Moroianu, Nearly Kähler manifolds with reduced holonomy, Ann. Global Anal. Geom., 19 (2001), pp. 307-319.

[4] D. BlaiR, Contact manifolds in Riemannian geometry, Lect. Notes Math., vol 509, Springer Verlag, 1976.

[5] J.-M. Bismut, A local index theorem for non-Kähler manifolds, Math. Ann., 284 (1989), pp. 681-699.

[6] C. Boyer, K. Galicki, M. Nakamaye, On the geometry of Sasakian-Einstein 5-manifolds, math.DG/0012047.

[7] D. Chinea, G. Gonzales, A classification of almost contact metric manifolds, Ann. di mat. Pura Appl., 156 (1990), pp. 15-36.

[8] D. Chinea, J.C. Marrero, Classifications of almost contact metric structures, Rev. Roumaine Math. Pures Appl., 37 (1992), pp. 199-212.

[9] L. Cordero, M. Fernandez, Some compact solvmanifolds of $G_{2}$-coassociative type, Analele Univ. Bucuresti, 36 (1987), pp. 16-19.

[10] P. Dalakov, S. Ivanov, Harmonic spinors of Dirac operator of connection with torsion in dimension 4, Class. Quantum Grav., 18 (2001), pp. 253-265.

[11] M. FERnANDEZ, A. GRAY, Riemannian manifolds with structure group $G_{2}$, Ann. Mat. Pura Appl., 132 (1982), pp. 19-45.

[12] M. Fernandez, L. Ugarte, Dolbeault cohomolgy for $G_{2}$-manifolds, Geom. Dedicata, 70 (1998), pp. 57-86.

[13] Th. FrIEDRICH, Der erste Eigenwert des Dirac-Operators einer kompakten Riemannschen Mannigfaltigkeit nichtnegativer Skalarkrümmung, Math. Nachr., 97 (1980), pp. 117-146.

[14] Th. Friedrich, Dirac Operators in Riemannian Geometry, Graduate Studies in Mathematics, vol. 25, AMS, 2000.

[15] Th. FrIEDRICH, Weak Spin(9)-structures on 16-dimensional Riemannian manifolds, Asian Math. Journ., 5 (2001), pp. 129-160.

[16] Th. Friedrich, R. Grunewald, On Einstein metrics on the twistor space of a fourdimensional Riemannian manifold, Math. Nachr., 123 (1985), pp. 55-60.

[17] Th. Friedrich, S. IVANov, Almost contact manifolds and type II string equations, math.dg/0111131.

[18] TH. FRIEDRICH, I. KATH, Einstein manifolds of dimension five with small eigenvalue of the Dirac operator, Journ. Diff. Geom., 29 (1989), pp. 263-279.

[19] Th. Friedrich, I. Kath, A. Moroianu, U. Semmelmann, On nearly parallel $G_{2}$-structures, Journ. Geom. Phys., 23 (1997), pp. 256-286.

[20] Th. Friedrich, E. C. KIM, The Einstein-Dirac equation on Riemannian spin manifolds, Journ. Geom. Phys., 33 (2000), pp. 128-172.

[21] Th. Friedrich, S. Sulanke, Ein Kriterium für die formale Selbstadjungiertheit des DiracOperators, Coll. Math. vol. XL (1979), pp. 239-247.

[22] W. Fulton, J. HARRIS, Representation theory, Springer-Verlag, 1991.

[23] S. GATES, C. Hull, M. Roč̌K, Twisted multiplets and new supersymmetric nonlinear sigma models, Nucl. Phys. B 248, pp. 157-186.

[24] P. Gauduchon, Structures de Weyl-Einstein, espaces de twisteurs et varietes de type $S^{1} \times S^{3}$, Journ. Reine Angew. Math., 469 (1995), pp. 1-50.

[25] P. Gauduchon, Hermitian connections and Dirac operators, Bol. U. M. I. ser. VII, vol. XI-B, supl. 2 (1997), pp. 257-289.

[26] J. Gauntlett, N. Kim, D. Martelli, D. Waldram, Fivebranes wrapped on SLAG threecycles and related geometry, JHEP, Vol. 11, 18 (2001).

[27] G.W. GibBons, G. PAPAdOPOUlOS, K.S. StElle, HKT and OKT geometries on soliton black hole moduli space, Nucl. Phys. B, 508 (1997), pp. 623-658.

[28] A. GraY, The structure of nearly Kähler manifolds, Math. Ann., 223 (1976), pp. 233-248.

[29] A. Gray, L. Hervella, The sixteen classes of almost hermitian manifolds and their linear invariants, Ann. di Mat. pura ed appl., 123 (1980), pp. 35-58.

[30] R. GRUnewald, Six-dimensional Riemannian manifolds with real Killing spinors, Ann. Global Anal. Geom., 8 (1990), pp. 43-59.

[31] R. GRUNEWALD, On the relation between real Killing spinors and almost hermitian structures, 
Preprint No. 271, Fachbereich Mathematik, Humboldt-Universität zu Berlin, 1991.

[32] S. Gubser, I.R. Klebanov, A.M. Polyakov, Gauge theory correlators from non-critical string theory, Phys. Lett., 428B (1998), pp. 105-114.

[33] N. Hitchin, Harmonic spinors, Adv. in Math., 14 (1974), pp. 1-55.

[34] P.S. Howe, G. PApadopoulos, Finiteness and anomalies in $(4,0)$ supersymmetric sigma models, Nucl. Phys. B, 381 (1992), pp. 360-372.

[35] P.S. Howe, G. PAPAdOPOulos, Twistor spaces for hyper-Kähler manifolds with torsion, Phys. Lett. B, 379 (1996), pp. 81-86.

[36] S. Ivanov, G. Papadopoulos, Vanishing theorems and string Backgrounds, Class. Quant. Grav., 18 (2001), pp. 1089-1110.

[37] V. Kirichenko, K-spaces of maximal rank: (russian), Mat. Zam., 22 (1977), pp. 465-476.

[38] O. KowAlski, S. WEgrzynOWSKI, A classification of five-dimensional $\varphi$-symmetric spaces, Tensor, N.S., 46 (1987), pp. 379-386.

[39] J.M. MALDACENA, The large $N$ limit of superconformal field theories and supergravity, Adv. Theor. Math. Phys., 2 (1998), pp. 231-252.

[40] B. McInnes, Spin holonomy of Einstein manifolds, Comm. Math. Phys., 203 (1999), pp. 349-364.

[41] A. MoRoIAnU, Kähler manifolds with small eigenvalues of the Dirac operator and a conjecture of Lichnerowicz, Ann. Inst. Fourier, 49 (1999), pp. 1637-1659.

[42] A. Opfermann, G. PAPAdopoulos, Homogeneous HKT and QKT manifolds, math$\mathrm{ph} / 9807026$.

[43] G. PAPAdOPOUlOS, Brane solitons and hypercomplex structures, math.DG/0003024.

[44] G. Papadopoulos, A. Teschendorff, Multi-angle five-brane intersections, Phys. Lett. B, 443 (1998), pp. 159-166.

[45] G. Papadopoulos, A. Teschendorff, Grassmannians, calibrations and five-brane intersections, Class. Quant. Grav., 17 (2000), pp. 2641-2662.

[46] E. SchröDINGER, Diracsches Elektron im Schwerfeld I, Sitzungsberichte der Preussischen Akademie der Wissenschaften Phys.-Math. Klasse 1932, Verlag der Akademie der Wissenschaften, Berlin 1932, pp. 436-460.

[47] P. Spindel, A. Sevrin, W. Troost, A. VA.: Proeyen, Extended supersymmetric $\sigma$-models on group manifolds, Nucl. Phys. B, 308 (1988),pp. 662-698.

[48] A. Strominger, Superstrings with torsion, Nucl. Phys. B, 274 (1986), pp. 253-284.

[49] A. Swann, Weakening holonomy, Preprint ESI No., 816 (2000).

[50] Y. McKenzie Wang, Parallel spinors and parallel forms, Ann. Global Anal. Geom., 7 (1989), pp. 59-68.

[51] E. Witten, Anti de sitter space and holography. Adv. Theor. Math. Phys., 2 (1998), pp. 253-291. 
